\title{
Pseudo almost periodic synchronization of Clifford-valued fuzzy cellular neural networks with time-varying delays on time scales
}

\author{
Yongkun $\mathrm{Li}^{1 *}$ (D) and Shiping Shen ${ }^{1}$
}

"Correspondence: yklie@ynu.edu.cn 1 Department of Mathematics, Yunnan University, Kunming, Yunnan 650091, China

\section{Springer}

\begin{abstract}
At present, the research on discrete-time Clifford-valued neural networks is rarely reported. However, the discrete-time neural networks are an important part of the neural network theory. Because the time scale theory can unify the study of discreteand continuous-time problems, it is not necessary to separately study continuousand discrete-time systems. Therefore, to simultaneously study the pseudo almost periodic oscillation and synchronization of continuous- and discrete-time Clifford-valued neural networks, in this paper, we consider a class of Clifford-valued fuzzy cellular neural networks on time scales. Based on the theory of calculus on time scales and the contraction fixed point theorem, we first establish the existence of pseudo almost periodic solutions of neural networks. Then, under the condition that the considered network has pseudo almost periodic solutions, by designing a novel state-feedback controller and using reduction to absurdity, we obtain that the drive-response structure of Clifford-valued fuzzy cellular neural networks on time scales with pseudo almost periodic coefficients can realize the global exponential synchronization. Finally, we give a numerical example to illustrate the feasibility of our results.
\end{abstract}

Keywords: Clifford-valued fuzzy cellular neural network; Pseudo almost periodic synchronization; Global exponential synchronization; Time scale

\section{Introduction}

Fuzzy cellular neural networks, introduced into the field of artificial neural networks in 1996 by Yang and Yang [1, 2], are a combination of fuzzy operations (fuzzy AND and fuzzy OR) and cellular neural networks. They combine the advantages of neural network and fuzzy theory and integrate learning, association, recognition, and information processing. Because fuzzy neural networks are based on uncertainty, which is a common problem in the study of brain model, they are closer to human brain than the general neural networks. Therefore the fuzzy cellular neural networks are widely used in the fields such as pattern recognition, computer science, artificial intelligence, optimal control, equation solving, robotics, military science, and so on. Because the application of neural networks in these fields is related to their long-term behaviors and the time delay is inevitable in real neural

(c) The Author(s) 2020. This article is licensed under a Creative Commons Attribution 4.0 International License, which permits use, sharing, adaptation, distribution and reproduction in any medium or format, as long as you give appropriate credit to the original author(s) and the source, provide a link to the Creative Commons licence, and indicate if changes were made. The images or other third party material in this article are included in the article's Creative Commons licence, unless indicated otherwise in a credit line to the material. If material is not included in the article's Creative Commons licence and your intended use is not permitted by statutory regulation or exceeds the permitted use, you will need to obtain permission directly from the copyright holder. To view a copy of this licence, visit http://creativecommons.org/licenses/by/4.0/. 
networks, the dynamics of fuzzy cellular neural networks with various time delays has been extensively studied [3-9].

On the one hand, it is well known that discrete- and continuous-time systems have the same importance in theory and practice, and the discrete-time systems are more convenient for calculation and numerical simulation. Therefore it is necessary to study the discrete-time systems while studying continuous-time systems. Fortunately, studying the neural network systems on time scales can unify the research of discrete- and continuoustime neural networks. So it is necessary and meaningful to study neural network models on time scales [10-16].

On the other hand, Clifford-valued neural network models are a kind of multidimensional neural network models, which are introduced into the study of neural networks in $[17,18]$. They include real-valued, complex-valued, and quaternion-valued neural network models as their particular cases. Because of the potential application value of Clifford-valued neural network models in high-dimensional data processing, they have attracted researchers' attention in recent years [17-23]. However, so far, there are few results on the dynamics of Clifford-valued neural networks [22-27]. In particular, up to date, there are no papers published on the dynamics of Clifford-valued fuzzy cellular neural networks on time scales.

Moreover, we know that periodic, almost periodic, and almost automorphic oscillations are important dynamics of nonautonomous neural networks. Pseudo almost periodicity is an extension of almost periodicity, so it is of great theoretical and practical significance to study the pseudo almost periodic oscillations of neural networks. Therefore many scholars have studied the pseudo almost periodic oscillation of neural networks [28-37]. In addition, synchronization is a common phenomenon in real systems, which shows that many different systems can adjust each other to produce a common dynamic behavior. With the help of synchronization, we can know the behavior of the unknown system through the known one. Therefore, as a powerful tool, it plays an important role in network control and system design. There are many results in the study of synchronization of neural networks [38-49]. However, the results of pseudo almost periodic synchronization of Clifford-valued neural networks on time scales have not been reported.

Based on the above observations and discussion, the primary purpose of this paper is to study the existence of pseudo almost periodic solutions and synchronization for fuzzy cellular neural networks with time-varying delays on time scales. This is the first paper studying the existence of pseudo almost periodic solutions and synchronization of Clifford-valued fuzzy cellular neural networks on time scales.

The remainder of the paper is organized as follows. In Sect. 2, we give some preliminaries and model description. In Sect. 3, we obtain sufficient conditions for the existence of pseudo almost periodic solutions of the proposed networks on time scales. In Sect. 4, we investigate global exponential synchronization. In Sect. 5, we show the effectiveness and feasibility of the developed methods in this paper by a numerical example. In Sect. 6, we draw a brief conclusion.

\section{Preliminaries and model description}

The real Clifford algebra over $\mathbb{R}^{m}$ is defined as

$$
\mathcal{A}=\left\{\sum_{A \subseteq\{1,2, \ldots, m\}} u^{A} e_{A}, u^{A} \in \mathbb{R}\right\},
$$


where $e_{A}=e_{h_{1}} e_{h_{2}} \cdots e_{h_{v}}$ with $A=\left\{h_{1}, h_{2}, \ldots, h_{v}\right\}, 1 \leq h_{1}<h_{2}<\cdots<h_{v} \leq m$. Moreover, $e_{\emptyset}=e_{0}=1$ and $e_{\{h\}}=e_{h}, h=1,2, \ldots, m$, are called Clifford generators, which satisfy the relations

$$
\begin{cases}e_{i}^{2}=1, & i=1,2, \ldots, s<m, \\ e_{i}^{2}=-1, & i=s+1, s+2, \ldots, m, \\ e_{i} e_{j}+e_{j} e_{i}=0, & i \neq j .\end{cases}
$$

Let $Q=\{\emptyset, 1,2, \ldots, A, \ldots, 12 \cdots m\}$. Then it is easy to see that $\mathcal{A}=\left\{\sum_{A} u^{A} e_{A}, u^{A} \in \mathbb{R}\right\}$, where $\sum_{A}$ is a shorthand for $\sum_{A \in Q}$. For $u=\sum_{A} u^{A} e_{A} \in \mathcal{A}$ and $v=\left(v_{1}, v_{2}, \ldots, v_{n}\right)^{T} \in \mathcal{A}^{n}$, the norms of $u$ and $v$ are defined as $\|u\|_{\mathcal{A}}=\sqrt{\sum_{A}\left(u^{A}\right)^{2}}$ and $\|v\|_{\mathcal{A}^{n}}=\max _{1 \leq p \leq n}\left\|v_{p}\right\|_{\mathcal{A}}$, respectively; for $v=\left(v_{1}, v_{2}, \ldots, v_{n}\right)^{T} \in \mathcal{A}^{n}$, the norm of $v$ is defined as $\|v\|_{\mathcal{A}^{n}}=\max _{1 \leq p \leq n}\left\|v_{p}\right\|_{\mathcal{A}}$. For information on the Clifford algebra, we refer the reader to [50].

Definition 2.1 ([8]) For $x, y \in \mathbb{R}$, we denote

$$
x \wedge y=\min \{x, y\} \quad \text { and } \quad x \vee y=\max \{x, y\} .
$$

For $x=\sum_{A \in Q} x^{A} e_{A}, y=\sum_{A \in Q} y^{A} e_{A} \in \mathcal{A}$, we denote $x \wedge y=\sum_{A \in Q}\left(x^{A} \wedge y^{A}\right) e_{A}$ and $x \vee y=$ $\sum_{A \in Q}\left(x^{A} \vee y^{A}\right) e_{A}$.

Let $\mathbb{T}$ be a time scale, that is, an arbitrary nonempty closed subset of the real set $\mathbb{R}$ with the topology and ordering inherited from $\mathbb{R}$, let $\sigma$ and $\eta$ denote the forward jump operator and the graininess function, respectively, and let $\mathcal{R}$ denote the set of regressive functions on $\mathbb{T}$. We define the set $\mathcal{R}^{+}=\{r \in \mathcal{R}: 1+\mu(t) r(t)>0, \forall t \in \mathbb{T}\}$. For the time scale theory, we refer the reader to [51].

Definition 2.2 Let $z=\sum_{A} z^{A} e_{A}: \mathbb{T} \rightarrow \mathcal{A}$, where $z^{A}: \mathbb{T} \rightarrow \mathbb{R}$. The delta derivative of the function $z$ is $z^{\Delta}(t)=\sum_{A \in Q}\left(z^{A}\right)^{\Delta}(t) e_{A}$, provided that $\left(z^{A}\right)^{\Delta}(t)$ exists for each $A \in Q$.

Definition 2.3 ([52]) A time scale $\mathbb{T}$ is called an almost periodic time scale if

$$
\Pi:=\{\tau \in \mathbb{R}: t \pm \tau \in \mathbb{T}, \forall t \in \mathbb{T}\} \neq\{0\}
$$

From now on, we assume that $\mathbb{T}$ is an almost periodic time scale.

Definition 2.4 A function $f \in C\left(\mathbb{T}, \mathcal{A}^{n}\right)$ is called almost periodic if for every $\varepsilon>0$, there exists a constant $l(\varepsilon)>0$ such that each interval of length $l(\varepsilon)$ contains $\tau \in \Pi$ such that

$$
\|f(t+\tau)-f(t)\|_{\mathcal{A}^{n}}<\varepsilon, \quad \forall t \in \mathbb{T} .
$$

We denote by $A P\left(\mathbb{T}, \mathcal{A}^{n}\right)$ the set of all almost periodic functions defined on $\mathbb{T}$ and by $B C\left(\mathbb{T}, \mathcal{A}^{n}\right)$ the set of all bounded continuous functions from $\mathbb{T}$ to $\mathcal{A}^{n}$. 
Let

$$
\begin{aligned}
\operatorname{PAP}_{0}\left(\mathbb{T}, \mathcal{A}^{n}\right)= & \left\{f \in B C\left(\mathbb{T}, \mathcal{A}^{n}\right): f \text { is } \Delta\right. \text {-measurable such that } \\
& \left.\lim _{r \rightarrow+\infty} \frac{1}{2 r} \int_{-r}^{r}\|f(s)\|_{\mathcal{A}^{n}} \Delta s=0, r \in \mathbb{T}\right\} .
\end{aligned}
$$

Inspired by Definition 3 in [11], we introduce the following definition.

Definition 2.5 A function $f \in B C\left(\mathbb{T}, \mathcal{A}^{n}\right)$ is called pseudo almost periodic if $f=g+h$, where $g \in A P\left(\mathbb{T}, \mathcal{A}^{n}\right)$ and $h \in P A P_{0}\left(\mathbb{T}, \mathcal{A}^{n}\right)$.

We denote by $P A P\left(\mathbb{T}, \mathcal{A}^{n}\right)$ the class of all pseudo almost periodic functions defined on $\mathbb{T}$.

From the above definition, similarly to the proofs of Lemmas 2.5 and 2.6 in [11], it is not difficult to prove the following two lemmas.

Lemma 2.1 If $\alpha \in \mathbb{R}, f, g \in P A P\left(\mathbb{T}, \mathcal{A}^{n}\right)$, then $\alpha f, f+g, f \times g \in P A P\left(\mathbb{T}, \mathcal{A}^{n}\right)$.

Lemma 2.2 If $\in C\left(\mathcal{A}, \mathcal{A}^{n}\right)$ satisfies the Lipschitz condition, $x \in P A P(\mathbb{T}, \mathcal{A}), \tau \in C^{1}(\mathbb{T}, \mathbb{R}) \cap$ $A P(\mathbb{T}, \Pi)$, and $\inf _{t \in \mathbb{T}}\left(1-\tau^{\Delta}(t)\right)>0$, then $f(x(\cdot-\tau(\cdot))) \in P A P\left(\mathbb{T}, \mathcal{A}^{n}\right)$.

The following lemma can be proved by the same proof method as that for Lemma 6 in [53].

Lemma 2.3 Let $a \in A P\left(\mathbb{T}, \mathbb{R}^{+}\right)$with $-a \in \mathcal{R}^{+}, \inf _{t \in \mathbb{T}} a(t)=a^{-}>0$, and $g \in P A P(\mathbb{T}, \mathcal{A})$. Then the function $F: t \rightarrow \int_{-\infty}^{t} e_{-a}(t, \sigma(s)) g(s) \Delta$ s belongs to $P A P(\mathbb{T}, \mathcal{A})$.

Similarly to the proof of Corollary 1 in [1], we can prove the following:

Lemma 2.4 Suppose that $\alpha_{i}, \beta_{i} \in C(T, \mathcal{A})$ and $f_{i} \in C(\mathcal{A}, \mathcal{A}), i=1,2, \ldots, n$. Then we have

$$
\begin{aligned}
& \left\|\bigwedge_{i=1}^{n} \alpha_{i} f_{i}(x)-\bigwedge_{i=1}^{n} \alpha_{i} f_{i}(y)\right\|_{\mathcal{A}} \leq \sum_{i=1}^{n}\left\|\alpha_{i}\right\|_{\mathcal{A}}\left\|f_{i}(x)-f_{i}(y)\right\|_{\mathcal{A}}, \quad i=1,2, \ldots, n, \\
& \left\|\bigvee_{i=1}^{n} \beta_{i} f_{i}(x)-\bigvee_{i=1}^{n} \beta_{i} f_{i}(y)\right\|_{\mathcal{A}} \leq \sum_{i=1}^{n}\left\|\beta_{i}\right\|_{\mathcal{A}}\left\|f_{i}(x)-f_{i}(y)\right\|_{\mathcal{A}}, \quad i=1,2, \ldots, n
\end{aligned}
$$

Similarly to the proof of Lemma 2.2 in [28], we can easily prove the following:

Lemma 2.5 If $\mathcal{F}_{i} \in \operatorname{PAP}(T, \mathcal{A}), i=1,2, \ldots, n$, then $\bigwedge_{i=1}^{n} \mathcal{F}_{i}(\cdot), \bigvee_{i=1}^{n} \mathcal{F}_{i}(\cdot) \in \operatorname{PAP}(T, \mathcal{A})$

In the paper, we consider the following Clifford-valued fuzzy cellular neural network with time-varying delays on time scale $\mathbb{T}$ :

$$
\begin{aligned}
x_{i}^{\Delta}(t)= & -a_{i}(t) x_{i}(t)+\sum_{j=1}^{n} b_{i j}(t) f_{j}\left(x_{j}\left(t-\eta_{i j}(t)\right)\right) \\
& +\bigwedge_{j=1}^{n} \alpha_{i j}(t) g_{j}\left(x_{j}\left(t-\tau_{i j}(t)\right)\right)+\bigvee_{j=1}^{n} \tilde{\alpha}_{i j}(t) \tilde{g}_{j}\left(x_{j}\left(t-\tilde{\tau}_{i j}(t)\right)\right)
\end{aligned}
$$




$$
\begin{aligned}
& +\sum_{j=1}^{n} d_{i j}(t) \mu_{j}(t)+\bigwedge_{j=1}^{n} T_{i j}(t) \mu_{j}(t) \\
& +\bigvee_{j=1}^{n} S_{i j}(t) \mu_{j}(t)+I_{i}(t), \quad i=1,2, \ldots, n,
\end{aligned}
$$

where $n$ is the number of neurons in layers; $x_{i}(t) \in \mathcal{A}, \mu_{j}(t) \in \mathcal{A}$, and $I_{i}(t) \in \mathcal{A}$ express the state, input, and bias of the $i$ th neuron, respectively, where $\mathcal{A}$ is a Clifford algebra; $a_{i}(t)>$ 0 is the rate at which the $i$ th neuron resets its potential to the resting state in isolation when they are disconnected from the network and the external inputs at time $t ; \alpha_{i j}(t) \in \mathcal{A}$ represents an element of the fuzzy feedback MIN template; $\tilde{\alpha}_{i j}(t) \in \mathcal{A}$ is an element of the fuzzy feedback MAX template; $T_{i j}(t) \in \mathcal{A}$ and $S_{i j}(t) \in \mathcal{A}$ are fuzzy feed forward MIN template and fuzzy feed forward MAX template, respectively; $b_{i j}(t) \in \mathcal{A}$ is an element of the feedback template; $d_{i j}(t) \in \mathcal{A}$ is the feed forward template; $\wedge$ and $\bigvee$ denote the fuzzy AND and fuzzy OR operations, respectively; $f_{j}, g_{j}$, and $\tilde{g}_{j}: \mathcal{A} \rightarrow \mathcal{A}$ are the activation functions; $\eta_{i j}(t), \tau_{i j}(t)$, and $\tilde{\tau}_{i j}(t)$ correspond to the transmission delays at time $t$ and satisfy $t-\eta_{i j}(t), t-\tau_{i j}(t)$, and $t-\tilde{\tau}_{i j}(t) \in \mathbb{T}$ for $t \in \mathbb{T}$.

Throughout the rest of the paper, we adopt the following notation:

$$
\begin{aligned}
& a_{i}^{-}=\inf _{t \in \mathbb{T}} a_{i}(t), \quad b_{i j}^{+}=\sup _{t \in \mathbb{T}}\left\|b_{i j}(t)\right\|_{\mathcal{A}}, \quad \alpha_{i j}^{+}=\sup _{t \in \mathbb{T}}\left\|\alpha_{i j}(t)\right\|_{\mathcal{A}}, \\
& \tilde{\alpha}_{i j}^{+}=\sup _{t \in \mathbb{T}}\left\|\tilde{\alpha}_{i j}(t)\right\|_{\mathcal{A}}, \quad \eta_{i j}^{+}=\sup _{t \in \mathbb{T}} \eta_{i j}(t), \quad \tau_{i j}^{+}=\sup _{t \in \mathbb{T}} \tau_{i j}(t), \\
& \tilde{\tau}_{i j}^{+}=\sup _{t \in \mathbb{T}} \tilde{\tau}_{i j}(t), \quad \mathcal{I}=\{1,2, \ldots, n\}, \quad \zeta=\max _{i, j \in \mathcal{I}}\left\{\eta_{i j}^{+}, \tau_{i j}^{+}, \tilde{\tau}_{i j}^{+}\right\} .
\end{aligned}
$$

The initial values of system (1) are as follows:

$$
x_{i}(s)=\varphi_{i}(s) \in \mathcal{A}, \quad s \in[-\zeta, 0]_{\mathbb{T}},
$$

where $\varphi_{i} \in C\left([-\zeta, 0]_{\mathbb{T}}, \mathcal{A}\right), i \in \mathcal{I}$.

To obtain our primary results, we need the following assumptions:

$\left(S_{1}\right)$ For $i, j \in \mathcal{I}, a_{i} \in A P\left(\mathbb{T}, \mathbb{R}^{+}\right)$with $-a_{i} \in \mathcal{R}^{+}, \eta_{i j}, \tau_{i j}, \tilde{\tau}_{i j} \in C^{1}\left(\mathbb{T}, \mathbb{R}^{+}\right) \cap A P(\mathbb{T}, \Pi)$ with $\inf _{t \in \mathbb{R}}\left\{\left(1-\eta_{i j}^{\Delta}(t)\right),\left(1-\tau_{i j}^{\Delta}(t)\right),\left(1-\tilde{\tau}_{i j}^{\Delta}(t)\right)\right\}>0$, and $b_{i j}, \alpha_{i j}, \tilde{\alpha}_{i j}, \mu_{j}, d_{i j}, S_{i j}, T_{i j}, I_{i} \in$ $\operatorname{PAP}(\mathbb{T}, \mathcal{A})$.

$\left(S_{2}\right)$ For $j \in \mathcal{I}, f_{j}, g_{j}, \tilde{g}_{j} \in C(\mathcal{A}, \mathcal{A})$, d there exist positive constants $L_{j}^{f}, L_{j}^{g}, L_{j}^{\tilde{g}}$ such that for any $u, v \in \mathcal{A},\left\|f_{j}(u)-f_{j}(v)\right\|_{\mathcal{A}} \leq L_{j}^{f}\|u-v\|_{\mathcal{A}},\left\|g_{j}(u)-g_{j}(v)\right\|_{\mathcal{A}} \leq L_{j}^{g}\|u-v\|_{\mathcal{A}}, \| \tilde{g}_{j}(u)-$ $\tilde{g}_{j}(v)\left\|_{\mathcal{A}} \leq L_{j}^{\tilde{g}}\right\| u-v \|_{\mathcal{A}}$.

$\left(S_{3}\right) \max _{i \in \mathcal{I}}\left\{\frac{\mathcal{P}_{i}}{a_{i}^{-}}\right\} \leq \frac{1}{2}$ and $\max _{i \in \mathcal{I}}\left\{\frac{\mathcal{Q}_{i}}{a_{i}^{-}}\right\}=: \kappa<1$, where for $i, j \in \mathcal{I}$,

$$
\begin{aligned}
\mathcal{P}_{i} & =\sum_{j=1}^{n} b_{i j}^{+}\left(L_{j}^{f}+\frac{1}{2}\right)+\sum_{j=1}^{n} \alpha_{i j}^{+}\left(L_{j}^{g}+\frac{1}{2}\right)+\sum_{j=1}^{n} \tilde{\alpha}_{i j}^{+}\left(L_{j}^{\tilde{g}}+\frac{1}{2}\right) \\
\mathcal{Q}_{i} & =\sum_{j=1}^{n} b_{i j}^{+} L_{j}^{f}+\sum_{j=1}^{n} \alpha_{i j}^{+} L_{j}^{g}+\sum_{j=1}^{n} \tilde{\alpha}_{i j}^{+} L_{j}^{\tilde{g}} .
\end{aligned}
$$




\section{The existence of pseudo almost periodic solutions}

In this section, we state and prove sufficient conditions for the existence of pseudo almost periodic solutions of (1).

Let $\mathbf{Y}=\operatorname{PAP}\left(\mathbb{T}, \mathcal{A}^{n}\right)$. Then it is a Banach space with the norm $\|f\|_{\mathbf{Y}}=\sup _{t \in \mathbb{T}}\|f(t)\|_{\mathcal{A}^{n}}$. Take

$$
\mathbf{Y}_{0}=\left\{\varphi \in \mathbf{Y} \mid\left\|\varphi-\varphi_{0}\right\|_{\mathbf{Y}} \leq \varpi\right\}
$$

where $\varphi_{0}=\left(\varphi_{0}^{1}, \varphi_{0}^{2}, \ldots, \varphi_{0}^{n}\right)^{T}$,

$$
\begin{aligned}
\varphi_{0}^{i}(t)= & \int_{-\infty}^{t} e_{-a_{i}}(t, \sigma(s))\left(\sum_{j=1}^{n} d_{i j}(s) \mu_{j}(s)+\bigwedge_{j=1}^{n} T_{i j}(s) \mu_{j}(s)\right. \\
& \left.+\bigvee_{j=1}^{n} S_{i j}(s) \mu_{j}(s)+I_{i}(s)\right) \Delta s, \quad i \in \mathcal{I},
\end{aligned}
$$

and

$$
\varpi \geq\left\{\left\|\varphi_{0}\right\|_{\mathbf{Y}}, \max _{j \in \mathcal{I}}\left\{\left\|f_{j}(0)\right\|_{\mathcal{A}}\right\}, \max _{j \in \mathcal{I}}\left\{\left\|g_{j}(0)\right\|_{\mathcal{A}}\right\}, \max _{j \in \mathcal{I}}\left\{\left\|\tilde{g}_{j}(0)\right\|_{\mathcal{A}}\right\}\right\} .
$$

Theorem 3.1 Let $\left(S_{1}\right)-\left(S_{3}\right)$ be satisfied. Then system (1) possesses only one pseudo almost periodic solution in $\mathbf{Y}_{0}$.

Proof Firstly, it is easy to check that if $x \in B C\left(\mathbb{T}, \mathcal{A}^{n}\right)$ is a solution of the integral equation

$$
\begin{aligned}
x_{i}(t)= & \int_{-\infty}^{t} e_{-a_{i}}(t, \sigma(s))\left(\sum_{j=1}^{n} b_{i j}(s) f_{j}\left(x_{j}\left(s-\eta_{i j}(s)\right)\right)\right. \\
& +\bigwedge_{j=1}^{n} \alpha_{i j}(s) g_{j}\left(x_{j}\left(s-\tau_{i j}(s)\right)\right)+\bigvee_{j=1}^{n} \tilde{\alpha}_{i j}(s) \tilde{g}_{j}\left(x_{j}\left(s-\tilde{\tau}_{i j}(s)\right)\right) \\
& +\sum_{j=1}^{n} d_{i j}(s) \mu_{j}(s)+\bigwedge_{j=1}^{n} T_{i j}(s) \mu_{j}(s) \\
& \left.+\bigvee_{j=1}^{n} S_{i j}(s) \mu_{j}(s)+I_{i}(s)\right) \Delta s, \quad i \in \mathcal{I},
\end{aligned}
$$

then $x$ is also a solution of system (1).

Secondly, we define the operator $\Upsilon: \mathbf{Y} \rightarrow B C\left(\mathbb{T}, \mathcal{A}^{n}\right)$ by

$$
\Upsilon \varphi=\left(\Upsilon_{1} \varphi, \Upsilon_{2} \varphi, \ldots, \Upsilon_{n} \varphi\right)^{T}
$$

where $\varphi \in \mathbf{Y}$,

$$
\left(\Upsilon_{i} \varphi\right)(t)=\int_{-\infty}^{t} e_{-a_{i}}(t, \sigma(s)) \mathcal{W}_{i}(s) \Delta s
$$


and

$$
\begin{aligned}
\mathcal{W}_{i}(s)= & \sum_{j=1}^{n} b_{i j}(s) f_{j}\left(\varphi_{j}\left(s-\eta_{i j}(s)\right)\right)+\bigwedge_{j=1}^{n} \alpha_{i j}(s) g_{j}\left(\varphi_{j}\left(s-\tau_{i j}(s)\right)\right) \\
& +\bigvee_{j=1}^{n} \tilde{\alpha}_{i j}(s) \tilde{g}_{j}\left(\varphi_{j}\left(s-\tilde{\tau}_{i j}(s)\right)\right)+\sum_{j=1}^{n} d_{i j}(s) \mu_{j}(s)+\bigwedge_{j=1}^{n} T_{i j}(s) \mu_{j}(s) \\
& +\bigvee_{j=1}^{n} S_{i j}(s) \mu_{j}(s)+I_{i}(s), \quad i \in \mathcal{I} .
\end{aligned}
$$

We will show that $\Upsilon: \mathbf{Y} \rightarrow \mathbf{Y}$ is well defined. In fact, by Lemmas 2.1, 2.2, and 2.5 for any $\varphi \in \mathbf{Y}$, we have $\mathcal{W}_{i}(s) \in P A P(\mathbb{T}, \mathcal{A}), i \in \mathcal{I}$. Furthermore, by Lemma 2.3 we conclude that $\Upsilon_{i} \varphi \in \operatorname{PAP}(\mathbb{T}, \mathcal{A}), i \in \mathcal{I}$. Hence $\Upsilon \varphi \in \mathbf{Y}$.

Thirdly, we will prove that $\Upsilon\left(\mathbf{Y}_{0}\right) \subset \mathbf{Y}_{0}$. In fact, for every $\varphi \in \mathbf{Y}_{0}$, we have that

$$
\|\varphi\|_{\mathbf{Y}} \leq\left\|\varphi-\varphi_{0}\right\|_{\mathbf{Y}}+\left\|\varphi_{0}\right\|_{\mathbf{Y}} \leq 2 \varpi
$$

and so we deduce that

$$
\begin{aligned}
\sup _{t \in \mathbb{T}} \| & \left(\Upsilon \varphi-\varphi_{0}\right)(t) \|_{\mathcal{A}^{n}} \\
= & \max _{i \in \mathcal{I}}\left\{\sup _{t \in \mathbb{T}} \| \int_{-\infty}^{t} e_{-a_{i}}(t, \sigma(s))\left(\sum_{j=1}^{n} b_{i j}(s) f_{j}\left(\varphi_{j}\left(s-\eta_{i j}(s)\right)\right)\right.\right. \\
& \left.\left.+\bigwedge_{j=1}^{n} \alpha_{i j}(s) g_{j}\left(\varphi_{j}\left(s-\tau_{i j}(s)\right)\right)+\bigvee_{j=1}^{n} \tilde{\alpha}_{i j}(s) \tilde{g}_{j}\left(\varphi_{j}\left(s-\tilde{\tau}_{i j}(s)\right)\right)\right) \Delta s \|_{\mathcal{A}}\right\} \\
\leq & \max _{i \in \mathcal{I}}\left\{\operatorname { s u p } _ { t \in \mathbb { T } } \left[\int _ { - \infty } ^ { t } e _ { - a _ { i } } ( t , \sigma ( s ) ) \left(\sum_{j=1}^{n} b_{i j}^{+}\left(L_{j}^{f}\left\|\varphi_{j}\left(s-\eta_{i j}(s)\right)\right\|_{\mathcal{A}}+\left\|f_{j}(0)\right\|_{\mathcal{A}}\right)\right.\right.\right. \\
& +\sum_{j=1}^{n} \alpha_{i j}^{+}\left(L_{j}^{g}\left\|\varphi_{j}\left(s-\tau_{i j}(s)\right)\right\|_{\mathcal{A}}+\left\|g_{j}(0)\right\|_{\mathcal{A}}\right) \\
& \left.\left.\left.+\sum_{j=1}^{n} \tilde{\alpha}_{i j}^{+}\left(L_{j}^{\tilde{g}}\left\|\varphi_{j}\left(s-\tilde{\tau}_{i j}(s)\right)\right\|_{\mathcal{A}}+\left\|\tilde{g}_{j}(0)\right\|_{\mathcal{A}}\right)\right) \Delta s\right]\right\} \\
\leq & \max _{i \in \mathcal{I}}\left\{\frac{2 \varpi}{a_{i}^{-}}\left(\sum_{j=1}^{n} b_{i j}^{+}\left(L_{j}^{f}+\frac{1}{2}\right)+\sum_{j=1}^{n} \alpha_{i j}^{+}\left(L_{j}^{g}+\frac{1}{2}\right)+\sum_{j=1}^{n} \tilde{\alpha}_{i j}^{+}\left(L_{j}^{\tilde{g}}+\frac{1}{2}\right)\right)\right\} \\
= & \max _{i \in \mathcal{I}}\left\{\frac{2 \varpi \mathcal{P}_{i}}{a_{i}^{-}}\right\},
\end{aligned}
$$

which, combined with condition $\left(S_{3}\right)$, implies that $\|\Phi \varphi\|_{\mathbf{Y}} \leq \varpi$. Hence $\Upsilon\left(\mathbf{Y}_{0}\right) \subset \mathbf{Y}_{0}$.

Finally, we will prove that $\Upsilon$ is a contraction. Noting that, for any $\varphi, \psi \in \mathbf{Y}_{0}$,

$$
\begin{aligned}
& \sup _{t \in \mathbb{T}}\|(\Upsilon \varphi-\Upsilon \psi)(t)\|_{\mathcal{A}^{n}} \\
& \quad=\max _{i \in \mathcal{I}}\left\{\sup _{t \in \mathbb{T}} \| \int_{-\infty}^{t} e_{-c_{i}}(t, \sigma(s))\left(\sum _ { j = 1 } ^ { n } b _ { i j } ( s ) \left(f_{j}\left(\varphi_{j}\left(s-\eta_{i j}(s)\right)\right)\right.\right.\right.
\end{aligned}
$$




$$
\begin{aligned}
& \left.-f_{j}\left(\psi_{j}\left(s-\eta_{i j}(s)\right)\right)\right)+\bigwedge_{j=1}^{n} \alpha_{i j}(s)\left(g_{j}\left(\varphi_{j}\left(s-\tau_{i j}(s)\right)\right)-g_{j}\left(\psi_{j}\left(s-\tau_{i j}(s)\right)\right)\right) \\
& \left.\left.+\bigvee_{j=1}^{n} \tilde{\alpha}_{i j}(s)\left(\tilde{g}_{j}\left(\varphi_{j}\left(s-\tilde{\tau}_{i j}(s)\right)\right)-\tilde{g}_{j}\left(\psi_{j}\left(s-\tilde{\tau}_{i j}(s)\right)\right)\right)\right) \Delta s \|_{\mathcal{A}}\right\} \\
\leq & \max _{i \in \mathcal{I}}\left\{\frac{1}{a_{i}^{-}}\left(\sum_{j=1}^{n} b_{i j}^{+} L_{j}^{f}+\sum_{j=1}^{n} \alpha_{i j}^{+} L_{j}^{g}+\sum_{j=1}^{n} \tilde{\alpha}_{i j}^{+} L_{j}^{\tilde{g}}\right)\right\}\|\varphi-\psi\|_{\mathbf{Y}} \\
= & \max _{i \in \mathcal{I}}\left\{\frac{\mathcal{Q}_{i}}{a_{i}^{-}}\right\}\|\varphi-\psi\|_{\mathbf{Y}},
\end{aligned}
$$

by $\left(S_{3}\right)$ we arrive at

$$
\|\Phi \varphi-\Phi \psi\|_{\mathbf{Y}} \leq \kappa\|\varphi-\psi\|_{\mathbf{Y}}
$$

Hence $\Upsilon$ is a contraction mapping. Consequently, $\Upsilon$ has a unique fixed point in $\mathbf{Y}_{0}$, that is, system (1) possesses a unique pseudo almost periodic solution in $\mathbf{Y}_{0}$. This completes the proof of Theorem 3.1.

\section{Pseudo almost periodic synchronization}

In this section, we take (1) as the driving system to study the global exponential synchronization of the drive-response structure of system (1). For this purpose, we take the following system as the response system:

$$
\begin{aligned}
y_{i}^{\Delta}(t)= & -a_{i}(t) y_{i}(t)+\sum_{j=1}^{n} b_{i j}(t) f_{j}\left(y_{j}\left(t-\eta_{i j}(t)\right)\right) \bigwedge_{j=1}^{n} \alpha_{i j}(t) g_{j}\left(y_{j}\left(t-\tau_{i j}(t)\right)\right) \\
& +\bigvee_{j=1}^{n} \tilde{\alpha}_{i j}(t) \tilde{g}_{j}\left(y_{j}\left(t-\tilde{\tau}_{i j}(t)\right)\right)+\sum_{j=1}^{n} d_{i j}(t) \mu_{j}(t)+\bigwedge_{j=1}^{n} T_{i j}(t) \mu_{j}(t) \\
& +\bigvee_{j=1}^{n} S_{i j}(t) \mu_{j}(t)+I_{i}(t)+\theta_{i}(t)
\end{aligned}
$$

where $t \in \mathbb{T}, i \in \mathcal{I}, y_{i}(t) \in \mathcal{A}$ denotes the state of the response system, $\theta_{i}(t)$ is a statefeedback controller, the remaining notations are the same as those in system (2), and the initial condition is as follows:

$$
y_{i}(s)=\psi_{i}(s) \in \mathcal{A}, \quad s \in[-\zeta, 0]_{\mathbb{T}},
$$

where $\psi_{i} \in C\left([-\zeta, 0]_{\mathbb{T}}, \mathcal{A}\right), i \in \mathcal{I}$.

Put $z_{i}(t)=y_{i}(t)-x_{i}(t)$. Subtracting (1) from (2) yields the following error system:

$$
\begin{aligned}
z_{i}^{\Delta}(t)= & -a_{i}(t) z_{i}(t)+\sum_{j=1}^{n} b_{i j}(t)\left(f_{j}\left(y_{j}\left(t-\eta_{i j}(t)\right)\right)-f_{j}\left(x_{j}\left(t-\eta_{i j}(t)\right)\right)\right) \\
& +\bigwedge_{j=1}^{n} \alpha_{i j}(s)\left(g_{j}\left(y_{j}\left(t-\tau_{i j}(t)\right)\right)-g_{j}\left(x_{j}\left(t-\tau_{i j}(t)\right)\right)\right)
\end{aligned}
$$




$$
+\bigvee_{j=1}^{n} \tilde{\alpha}_{i j}(t)\left(\tilde{g}_{j}\left(y_{j}\left(t-\tilde{\tau}_{i j}(t)\right)\right)-\tilde{g}_{j}\left(x_{j}\left(t-\tilde{\tau}_{i j}(t)\right)\right)\right)+\theta_{i}(t), \quad i \in \mathcal{I}
$$

To achieve the global exponential synchronization of the drive-response systems, we design the following state-feedback controller:

$$
\begin{aligned}
\theta_{i}(t)= & -c_{i}(t) z_{i}(t)+\bigwedge_{j=1}^{n} \beta_{i j}(t)\left(h_{j}\left(y_{j}\left(t-\tau_{i j}(t)\right)\right)-h_{j}\left(x_{j}\left(t-\tau_{i j}(t)\right)\right)\right) \\
& +\bigvee_{j=1}^{n} \tilde{\beta}_{i j}(t)\left(\tilde{h}_{j}\left(y_{j}\left(t-\tilde{\tau}_{i j}(t)\right)\right)-\tilde{h}_{j}\left(x_{j}\left(t-\tilde{\tau}_{i j}(t)\right)\right)\right), \quad i \in \mathcal{I} .
\end{aligned}
$$

Definition 4.1 The response system (2) and the drive system (1) are said to be globally exponentially synchronized if for every solution $z$ of the error system (3), there exist positive constants $\xi$ with $\ominus \xi \in \mathcal{R}^{+}$and $\mathcal{M}>1$ such that

$$
\|z(t)\|_{\mathcal{A}^{n}} \leq \mathcal{M}\|\psi-\varphi\|_{0} e_{\ominus \xi}\left(t, t_{0}\right), \quad t \in[0,+\infty)_{\mathbb{T}}
$$

where $t_{0} \in[-\zeta, 0]_{\mathbb{T}},\|\psi-\varphi\|_{0}=\sup _{s \in[-\zeta, 0]_{\mathbb{T}}}\|\psi(s)-\varphi(s)\|_{\mathcal{A}^{n}}$, and $\xi, M$ are independent of $z$.

Theorem 4.1 Let $\left(S_{1}\right)-\left(S_{3}\right)$ hold. Suppose further that:

$\left(S_{4}\right)$ For $i, j \in \mathcal{I}, c_{i} \in A P\left(\mathbb{T}, \mathbb{R}^{+}\right)$with $-\left(c_{i}+a_{i}\right) \in \mathcal{R}^{+}, \beta_{i j}, \tilde{\beta}_{i j} \in \operatorname{PAP}(\mathbb{T}, \mathcal{A})$.

$\left(S_{5}\right)$ For $i, j \in \mathcal{I}, h_{j}, \tilde{h}_{j} \in C(\mathcal{A}, \mathcal{A})$, and there exist positive constant numbers $L_{j}^{h}, L_{j}^{\tilde{h}}$ such that for any $u, v \in \mathcal{A}$,

$$
\left\|h_{j}(u)-h_{j}(v)\right\|_{\mathcal{A}} \leq L_{j}^{h}\|u-v\|_{\mathcal{A}}, \quad\left\|\tilde{h}_{j}(u)-\tilde{h}_{j}(v)\right\|_{\mathcal{A}} \leq L_{j}^{\tilde{h}}\|u-v\|_{\mathcal{A}} .
$$

$\left(S_{6}\right) \max _{i \in \mathcal{I}}\left\{\frac{\tilde{\mathcal{Q}}_{i}}{a_{i}^{-}+c_{i}^{-}}\right\}<1$, where $\tilde{\mathcal{Q}}_{i}=\mathcal{Q}_{i}+\sum_{j=1}^{n} \beta_{i j}^{+} L_{j}^{h}+\sum_{j=1}^{n} \tilde{\beta}_{i j}^{+} L_{j}^{\tilde{h}}$.

Then the response system (1) and the drive system (2) are globally exponentially synchronized.

Proof Multiplying (3) by $e_{-\left(a_{i}+c_{i}\right)}\left(t_{0}, \sigma(t)\right)$ and then integrating it over the interval $\left[t_{0}, t\right]_{\mathbb{T}}$, where $t_{0} \in[-\zeta, 0]_{\mathbb{T}}$, we get that

$$
\begin{aligned}
z_{i}(t)= & z_{i}\left(t_{0}\right) e_{-\left(a_{i}+c_{i}\right)}\left(t, t_{0}\right)+\int_{t_{0}}^{t} e_{-\left(a_{i}+c_{i}\right)}(t, \sigma(s)) \\
& \times\left(\sum_{j=1}^{n} b_{i j}(t)\left(f_{j}\left(y_{j}\left(s-\eta_{i j}(s)\right)\right)-f_{j}\left(x_{j}\left(s-\eta_{i j}(s)\right)\right)\right)\right. \\
& +\bigwedge_{j=1}^{n} \alpha_{i j}(s)\left(g_{j}\left(y_{j}\left(s-\tau_{i j}(s)\right)\right)-g_{j}\left(x_{j}\left(s-\tau_{i j}(s)\right)\right)\right) \\
& +\bigvee_{j=1}^{n} \tilde{\alpha}_{i j}(s)\left(\tilde{g}_{j}\left(y_{j}\left(s-\tilde{\tau}_{i j}(s)\right)\right)-\tilde{g}_{j}\left(x_{j}\left(s-\tilde{\tau}_{i j}(s)\right)\right)\right) \\
& +\bigwedge_{j=1}^{n} \beta_{i j}(s)\left(h_{j}\left(y_{j}\left(s-\tau_{i j}(s)\right)\right)-h_{j}\left(x_{j}\left(s-\tau_{i j}(s)\right)\right)\right)
\end{aligned}
$$




$$
\left.+\bigvee_{j=1}^{n} \tilde{\beta}_{i j}(s)\left(\tilde{h}_{j}\left(y_{j}\left(s-\tilde{\tau}_{i j}(s)\right)\right)-\tilde{h}_{j}\left(x_{j}\left(s-\tilde{\tau}_{i j}(s)\right)\right)\right)\right) \Delta s, \quad i \in \mathcal{I}
$$

Set

$$
\begin{aligned}
\Theta_{i}(\omega)= & a_{i}^{-}+c_{i}^{-}-\omega-\exp \left(\omega \sup _{s \in \mathbb{T}} \mu(s)\right)\left(\sum_{j=1}^{n} b_{i j}^{+} L_{j}^{f} \exp \left(\omega \eta_{i j}^{+}\right)\right. \\
& +\sum_{j=1}^{n}\left(\alpha_{i j}^{+} L_{j}^{g}+\beta_{i j}^{+} L_{j}^{h}\right) \exp \left(\omega \tau_{i j}^{+}\right) \\
& \left.+\sum_{j=1}^{n}\left(\tilde{\alpha}_{i j}^{+} L_{j}^{\tilde{g}}+\tilde{\beta}_{i j}^{+} L_{j}^{\tilde{h}}\right) \exp \left(\omega \tilde{\tau}_{i j}^{+}\right)\right), \quad i \in \mathcal{I} .
\end{aligned}
$$

Then by $\left(S_{6}\right)$, for $i \in \mathcal{I}$, we find

$$
\Theta_{i}(0)=a_{i}^{-}+c_{i}^{-}-\tilde{\mathcal{Q}}_{i}>0, \quad i \in \mathcal{I} .
$$

Because of the continuity of $\Theta_{i}$ and the fact that $\Theta_{i}(\omega) \rightarrow-\infty$ as $\omega \rightarrow+\infty$, we see that there exist $\theta_{i}$ such that $\Theta_{i}\left(\theta_{i}\right)=0$ and $\Theta_{i}(\omega)>0$ for $\omega \in\left(0, \theta_{i}\right), i \in \mathcal{I}$. Obviously, we have $\Theta_{i}(e) \geq 0, i \in \mathcal{I}$, where $e=\min _{i \in \mathcal{I}}\left\{\theta_{i}\right\}$. So, we can choose a positive constant $0<\xi<\min \left\{e, \min _{i \in \mathcal{I}}\left\{a_{i}^{-}+c_{i}^{-}\right\}\right\}$with $\ominus \xi \in \mathcal{R}^{+}$such that $\Theta_{i}(\xi)>0, i \in \mathcal{I}$, which implies that

$$
\begin{aligned}
& \frac{\exp \left(\xi \sup _{s \in \mathbb{T}} \mu(s)\right)}{a_{i}^{-}+c_{i}^{-}-\xi}\left(\sum_{j=1}^{n} b_{i j}^{+} L_{j}^{f} \exp \left(\xi \eta_{i j}^{+}\right)+\sum_{j=1}^{n}\left(\alpha_{i j}^{+} L_{j}^{g}+\beta_{i j}^{+} L_{j}^{h}\right) \exp \left(\xi \tau_{i j}^{+}\right)\right. \\
& \left.+\sum_{j=1}^{n}\left(\tilde{\alpha}_{i j}^{+} L_{j}^{\tilde{g}}+\tilde{\beta}_{i j}^{+} L_{j}^{\tilde{h}}\right) \exp \left(\xi \tilde{\tau}_{i j}^{+}\right)\right)<1, \quad i \in \mathcal{I} .
\end{aligned}
$$

Taking

$$
\mathcal{M}=\max _{i \in \mathcal{I}}\left\{\frac{a_{i}^{-}+c_{i}^{-}}{\tilde{\mathcal{Q}}_{i}}\right\}
$$

from $\left(S_{6}\right)$ we have $\mathcal{M}>1$. Thus

$$
\begin{aligned}
\frac{1}{\mathcal{M}}< & \frac{\exp \left(\xi \sup _{s \in \mathbb{T}} \mu(s)\right)}{a_{i}^{-}+c_{i}^{-}-\xi}\left(\sum_{j=1}^{n} b_{i j}^{+} L_{j}^{f} \exp \left(\xi \eta_{i j}^{+}\right)\right. \\
& \left.+\sum_{j=1}^{n}\left(\alpha_{i j}^{+} L_{j}^{g}+\beta_{i j}^{+} L_{j}^{h}\right) \exp \left(\xi \tau_{i j}^{+}\right)+\sum_{j=1}^{n}\left(\tilde{\alpha}_{i j}^{+} L_{j}^{\tilde{g}}+\tilde{\beta}_{i j}^{+} L_{j}^{\tilde{h}}\right) \exp \left(\xi \tilde{\tau}_{i j}^{+}\right)\right) .
\end{aligned}
$$

For $e_{\ominus \xi}\left(t, t_{0}\right)>1$, where $t \in\left[-\zeta, t_{0}\right]_{\mathbb{T}}$, it is evident that

$$
\|z(t)\|_{\mathcal{A}^{n}} \leq \mathcal{M} e_{\ominus \xi}\left(t, t_{0}\right)\|\psi-\varphi\|_{0}, \quad \forall t \in\left[-\zeta, t_{0}\right]_{\mathbb{T}} .
$$

Further, we will prove the following inequality:

$$
\|z(t)\|_{\mathcal{A}^{n}} \leq \mathcal{M} e_{\ominus \xi}\left(t, t_{0}\right)\|\psi-\varphi\|_{0}, \quad \forall t \in\left(t_{0},+\infty\right)_{\mathbb{T}} .
$$


To this end, we first prove that for any $\varsigma>1$,

$$
\|z(t)\|_{\mathcal{A}^{n}}<\varsigma \mathcal{M} e_{\ominus \xi}\left(t, t_{0}\right)\|\psi-\varphi\|_{0}, \quad \forall t \in\left(t_{0},+\infty\right)_{\mathbb{T}}
$$

which implies that for all $i \in \mathcal{I}$,

$$
\left\|z_{i}(t)\right\|_{\mathcal{A}}<\varsigma \mathcal{M} e_{\ominus \xi}\left(t, t_{0}\right)\|\psi-\varphi\|_{0}, \quad \forall t \in\left(t_{0},+\infty\right)_{\mathbb{T}} .
$$

Otherwise, if (7) is not true, then there exist $i_{0} \in \mathcal{I}$ and $\tilde{t} \in\left(t_{0},+\infty\right)_{\mathbb{T}}$ such that

$$
\left\|z_{i_{0}}(\tilde{t})\right\|_{\mathcal{A}} \geq \varsigma \mathcal{M}\|\psi-\varphi\|_{0} e_{\ominus \xi}\left(\tilde{t}, t_{0}\right)
$$

and

$$
\left\|z_{i_{0}}(t)\right\|_{\mathcal{A}}<\varsigma \mathcal{M}\|\psi-\varphi\|_{0} e_{\ominus \xi}\left(t, t_{0}\right), \quad t \in\left(t_{0}, \tilde{t}\right)_{\mathbb{T}} .
$$

Therefore there must exist a constant $C \geq 1$ such that

$$
\left\|z_{i_{0}}(\tilde{t})\right\|_{\mathcal{A}}=C_{\zeta} \mathcal{M}\|\psi-\varphi\|_{0} e_{\ominus \xi}\left(\tilde{t}, t_{0}\right)
$$

and

$$
\left\|z_{i_{0}}(t)\right\|_{\mathcal{A}}<C_{\zeta} \mathcal{M}\|\psi-\varphi\|_{0} e_{\ominus \xi}\left(t, t_{0}\right), \quad t \in\left(t_{0}, \tilde{t}\right)_{\mathbb{T}} .
$$

In view of (8), (9), (4), and $\mathcal{M}>1$, we have

$$
\begin{aligned}
& \left\|z_{i_{0}}(\tilde{t})\right\|_{\mathcal{A}} \\
& =\| z_{i_{0}}\left(t_{0}\right) e_{-\left(a_{i_{0}}+c_{i_{0}}\right)}\left(\tilde{t}, t_{0}\right)+\int_{t_{0}}^{\tilde{t}} e_{-\left(a_{i_{0}}+c_{i_{0}}\right)}(\tilde{t}, \sigma(s)) \\
& \quad \times\left(\sum_{j=1}^{n} b_{i_{0} j}(s)\left(f_{j}\left(y_{j}\left(s-\eta_{i_{0} j}(s)\right)\right)-f_{j}\left(x_{j}\left(s-\eta_{i_{j} j}(s)\right)\right)\right)\right. \\
& \quad+\bigwedge_{j=1}^{n} \alpha_{i_{0} j}(s)\left(g_{j}\left(\varphi_{j}\left(s-\tau_{i_{0} j}(s)\right)\right)-g_{j}\left(\psi_{j}\left(s-\tau_{i_{0} j}(s)\right)\right)\right) \\
& \quad+\bigvee_{j=1}^{n} \tilde{\alpha}_{i_{0} j}(s)\left(\tilde{g}_{j}\left(\varphi_{j}\left(s-\tilde{\tau}_{i_{0} j}(s)\right)\right)-\tilde{g}_{j}\left(\psi_{j}\left(s-\tilde{\tau}_{i_{0} j}(s)\right)\right)\right) \\
& \quad+\bigwedge_{j=1}^{n} \beta_{i_{0} j}(s)\left(h_{j}\left(\varphi_{j}\left(s-\tau_{i_{0} j}(s)\right)\right)-h_{j}\left(\psi_{j}\left(s-\tau_{i_{0} j}(s)\right)\right)\right) \\
& \left.\quad+\bigvee_{j=1}^{n} \tilde{\beta}_{i_{0 j} j}(s)\left(\tilde{h}_{j}\left(\varphi_{j}\left(s-\tilde{\tau}_{i_{0} j}(s)\right)\right)-\tilde{h}_{j}\left(\psi_{j}\left(s-\tilde{\tau}_{i_{0} j}(s)\right)\right)\right)\right) \Delta s \|_{\mathcal{A}} \\
& <\left\|z_{i_{0}}\left(t_{0}\right)\right\|\left\|_{\mathcal{A}} e_{-\left(a_{i_{0}}+c_{i_{0}}\right)}\left(\tilde{t}, t_{0}\right)+C \varsigma \mathcal{M}\right\| \psi-\varphi \|_{0} e_{\ominus \xi}\left(\tilde{t}, t_{0}\right) \\
& \quad \times \int_{t_{0}}^{\tilde{t}} e_{-\left(a_{i_{0}}+c_{i_{0}}\right)}(\tilde{t}, \sigma(s)) e_{\xi}(\tilde{t}, \sigma(s))\left(\sum_{j=1}^{n} b_{i_{0} j}^{+} L_{j}^{f} e_{\xi}\left(\sigma(s), s-\eta_{i_{0} j}(s)\right)\right.
\end{aligned}
$$




$$
\begin{aligned}
& +\sum_{j=1}^{n}\left(\alpha_{i_{0} j}^{+} L_{j}^{g}+\beta_{i_{0} j}^{+} L_{j}^{h}\right) e_{\xi}\left(\sigma(s), s-\tau_{i_{0} j}(s)\right) \\
& \left.+\sum_{j=1}^{n}\left(\tilde{\alpha}_{i_{0} j}^{+} L_{j}^{\tilde{g}}+\tilde{\beta}_{i_{0} j}^{+} \tilde{L}_{j}^{\tilde{h}}\right) e_{\xi}\left(\sigma(s), s-\tilde{\tau}_{i_{0} j}(s)\right)\right) \Delta s \\
& =\left\|z_{i_{0}}\left(t_{0}\right)\right\|_{\mathcal{A}} e_{-\left(a_{i_{0}}+c_{i_{0}}\right)}\left(\tilde{t}, t_{0}\right)+C_{\zeta} \mathcal{M}\|\psi-\varphi\|_{0} e_{\ominus \xi}\left(\tilde{t}, t_{0}\right) \\
& \times \int_{t_{0}}^{\tilde{t}} e_{-\left(a_{i_{0}}+c_{i_{0}}\right) \oplus \xi}(\tilde{t}, \sigma(s))\left(\sum_{j=1}^{n} b_{i_{0} j}^{+} L_{j}^{f} e_{\xi}\left(\sigma(s), s-\eta_{i_{0} j}(s)\right)\right. \\
& +\sum_{j=1}^{n}\left(\alpha_{i_{0} j}^{+} L_{j}^{g}+\beta_{i_{0} j}^{+} L_{j}^{h}\right) e_{\xi}\left(\sigma(s), s-\tau_{i_{0} j}(s)\right) \\
& \left.+\sum_{j=1}^{n}\left(\tilde{\alpha}_{i_{0} j}^{+} L_{j}^{\tilde{g}}+\tilde{\beta}_{i_{0} j}^{+} \tilde{L}_{j}^{\tilde{h}}\right) e_{\xi}\left(\sigma(s), s-\tilde{\tau}_{i_{0} j}(s)\right)\right) \Delta s \\
& \leq\left\|z_{i_{0}}\left(t_{0}\right)\right\|_{\mathcal{A}} e_{-\left(a_{i_{0}}+c_{i_{0}}\right)}\left(\tilde{t}, t_{0}\right)+C_{\zeta} \mathcal{M}\|\psi-\varphi\|_{0} e_{\ominus \xi}\left(\tilde{t}, t_{0}\right) \\
& \times \int_{t_{0}}^{\tilde{t}} e_{-\left(a_{i_{0}}+c_{i_{0}}\right) \oplus \xi}(\tilde{t}, \sigma(s))\left(\sum_{j=1}^{n} b_{i_{0}}^{+} L_{j}^{f} \exp \left(\xi\left(\eta_{i_{0} j}^{+}+\sup _{s \in \mathbb{T}} \mu(s)\right)\right)\right. \\
& +\sum_{j=1}^{n}\left(\alpha_{i_{0} j}^{+} L_{j}^{g}+\beta_{i_{0} j}^{+} L_{j}^{h}\right) \exp \left(\xi\left(\tau_{i_{0} j}^{+}+\sup _{s \in \mathbb{T}} \mu(s)\right)\right) \\
& \left.+\sum_{j=1}^{n}\left(\tilde{\alpha}_{i_{0} j}^{+} j_{j}^{\tilde{g}}+\tilde{\beta}_{i_{0} j}^{+} L_{j}^{\tilde{h}}\right) \exp \left(\xi\left(\tilde{\tau}_{i_{0} j}^{+}+\sup _{s \in \mathbb{T}} \mu(s)\right)\right)\right) \Delta s \\
& \leq C_{\zeta} \mathcal{M}\|\psi-\varphi\|_{0}\left\{\frac{e_{-\left(a_{i_{0}}+c_{i_{0}}\right) \oplus \xi}\left(\tilde{t}, t_{0}\right)}{C_{\zeta} \mathcal{M}}+\exp \left(\xi \sup _{s \in \mathbb{T}} \mu(s)\right)\right. \\
& \times\left(\sum_{j=1}^{n} b_{i_{0} j}^{+} L_{j}^{f} \exp \left(\xi \eta_{i_{0} j}^{+}\right)+\sum_{j=1}^{n}\left(\alpha_{i_{0}}^{+} L_{j}^{g}+\beta_{i_{0} j}^{+} L_{j}^{h}\right) \exp \left(\xi \tau_{i_{0}}^{+}\right)\right. \\
& \left.+\sum_{j=1}^{n}\left(\tilde{\alpha}_{i_{0} j}^{+} L_{j}^{\tilde{g}}+\tilde{\beta}_{i_{0} j}^{+} L_{j}^{\tilde{h}}\right) \exp \left(\xi \tilde{\tau}_{i_{0} j}^{+}\right)\right) \\
& \left.\times \int_{t_{0}}^{\tilde{t}} e_{-\left(a_{i_{0}}+c_{i_{0}}\right) \oplus \xi}(\tilde{t}, \sigma(s)) \Delta s\right\} e_{\ominus \xi}\left(\tilde{t}, t_{0}\right) \\
& \leq\left\{\frac{e_{-\left(a_{i_{0}}+c_{i_{0}}\right) \oplus \xi}\left(\tilde{t}, t_{0}\right)}{C_{S} \mathcal{M}}+\exp \left(\xi \sup _{s \in \mathbb{T}} \mu(s)\right)\left(\sum_{j=1}^{n} b_{i_{0}}^{+} L_{j}^{f} \exp \left(\xi \eta_{i_{0} j}^{+}\right)\right.\right. \\
& \left.+\sum_{j=1}^{n}\left(\alpha_{i_{0} j}^{+} L_{j}^{g}+\beta_{i_{0} j}^{+} L_{j}^{h}\right) \exp \left(\xi \tau_{i_{0} j}^{+}\right)+\sum_{j=1}^{n}\left(\tilde{\alpha}_{i_{0} j}^{+} L_{j}^{\tilde{g}}+\tilde{\beta}_{i_{0} j}^{+} L_{j}^{\tilde{h}}\right) \exp \left(\xi \tilde{\tau}_{i_{0} j}^{+}\right)\right) \\
& \left.\times \frac{1-e_{-\left(a_{i_{0}}+c_{i_{0}}\right) \oplus \xi}\left(\tilde{t}, t_{0}\right)}{a_{i_{0}}^{-}+c_{i_{0}}^{-}-\xi}\right\} C_{S} \mathcal{M}\|\psi-\varphi\|_{0} e_{\ominus \xi}\left(\tilde{t}, t_{0}\right) \\
& <\left\{\left[\frac{1}{\mathcal{M}}-\frac{\exp \left(\xi \sup _{s \in \mathbb{T}} \mu(s)\right)}{a_{i_{0}}^{-}+c_{i_{0}}^{-}-\xi}\left(\sum_{j=1}^{n} b_{i_{0}}^{+} L_{j}^{f} \exp \left(\xi \eta_{i_{0}}^{+}\right)\right.\right.\right.
\end{aligned}
$$




$$
\begin{aligned}
& \left.\left.+\sum_{j=1}^{n}\left(\alpha_{i_{0} j}^{+} L_{j}^{g}+\beta_{i_{0}}^{+} L_{j}^{h}\right) \exp \left(\xi \tau_{i_{0} j}^{+}\right)+\sum_{j=1}^{n}\left(\tilde{\alpha}_{i_{0} j}^{+} L_{j}^{\tilde{g}}+\tilde{\beta}_{i_{0} j}^{+} L_{j}^{\tilde{h}}\right) \exp \left(\xi \tilde{\tau}_{i_{0} j}^{+}\right)\right)\right] \\
& \times e_{-\left(a_{i_{0}}+c_{i_{0}}\right) \oplus \xi}\left(\tilde{t}, t_{0}\right)+\frac{\exp \left(\xi \sup _{s \in \mathbb{T}} \mu(s)\right)}{a_{i_{0}}^{-}+c_{i_{0}}^{-}-\xi} \\
& \times\left(\sum_{j=1}^{n} b_{i_{0} j}^{+} L_{j}^{f} \exp \left(\xi \eta_{i_{0} j}^{+}\right)+\sum_{j=1}^{n}\left(\alpha_{i_{0} j}^{+} L_{j}^{g}+\beta_{i_{0} j}^{+} L_{j}^{h}\right) \exp \left(\xi \tau_{i_{0} j}^{+}\right)\right. \\
& \left.\left.+\sum_{j=1}^{n}\left(\tilde{\alpha}_{i_{0} j}^{+} L_{j}^{\tilde{g}}+\tilde{\beta}_{i_{0}}^{+} L_{j}^{\tilde{h}}\right) \exp \left(\xi \tilde{\tau}_{i_{0} j}^{+}\right)\right)\right\} C \varsigma \mathcal{M}\|\psi-\varphi\|_{0} e_{\ominus \xi}\left(\tilde{t}, t_{0}\right) \\
& <C \varsigma \mathcal{M}\|\psi-\varphi\|_{0} e_{\ominus \xi}\left(\tilde{t}, t_{0}\right),
\end{aligned}
$$

which contradicts (8), and so (7) holds. Letting $\varsigma \rightarrow 1$, we conclude that (5) holds. As a result, the response system (1) and the drive system (2) are globally exponentially synchronized. The proof of Theorem 4.1 is completed.

\section{Examples}

In this section, we present an example to illustrate the feasibility and effectiveness of our results obtained in Sect. 4.

Example 5.1 In systems (1) and (2), let $m=3$ and $n=2$ and take the coefficients are follows:

$$
\begin{aligned}
a_{1}(t)= & 0.35+0.3|\cos \sqrt{3} t|, \quad a_{2}(t)=0.4+0.1|\sin 3 t|, \\
c_{1}(t)= & 0.6+0.1|\sin \sqrt{5} t|, \quad c_{2}(t)=0.3+0.2|\cos 5 t|, \\
f_{j}(x)= & \frac{1}{320} e_{0} \cos \sqrt{5} x^{13}+\frac{3}{800} \sin \left(x^{12}+x^{123}\right) e_{1}+\frac{1}{530} e_{2} \tanh 4 x^{13} \\
& +\frac{1}{390} e_{3} \cos \left(x^{2}+x^{13}\right)+\frac{1}{245} e_{12} \sin ^{2} x^{3}+\frac{1}{350} \cos \left(x^{3}+x^{1}\right) e_{13} \\
& +\frac{1}{390} e_{23} \tanh x^{12}+\frac{1}{380} e_{123} \sin \left(x^{0}+x^{2}+x^{23}\right), \quad j=1,2, \\
g_{j}(x)= & \tilde{g}_{j}(x)=\frac{\sqrt{3}}{480} e_{0} \cos \sqrt{3} x^{3}+\frac{1}{225}\left|x^{1}+x^{3}\right| e_{1}+\frac{1}{410} e_{2} \tanh x^{123} \\
& +\frac{2}{325} e_{3} \cos \left(x^{13}+x^{23}\right)+\frac{1}{430}\left(\left|x^{13}+1\right|-\left|x^{2}-1\right|\right) e_{12} \\
& +\frac{\sqrt{2}}{570} e_{13} \sin \left(x^{2}+x^{13}\right)+\frac{1}{485} e_{23} \cos x^{23} \\
& +\frac{1}{390} e_{123} \tanh \left(x^{1}+x^{12}+x^{13}\right), \quad j=1,2, \\
h_{j}(x)= & \tilde{h}_{j}(x)=\frac{\sqrt{3}}{1110} e_{0} \cos \sqrt{5}\left(x^{2}+x^{13}\right)+\frac{1}{345}\left|x^{12}+x^{123}\right| e_{1} \\
& +\frac{1}{530} e_{2} \tanh x^{2}+\frac{3}{870} e_{3} \sin \left(x^{23}+x^{2}\right)+\frac{1}{470} \cos \left(x^{12}\right) e_{12} \\
& +e_{13} \frac{\sqrt{3}}{990} \sin \left(x^{0}+x^{12}\right)+e_{23} \frac{1}{435} \tanh x^{23}
\end{aligned}
$$




$$
\begin{aligned}
& +\frac{1}{340} e_{123} \cos \left(x^{0}+x^{3}+x^{123}\right), \quad j=1,2, \\
& I_{1}(t)=0.4 e_{0} \cos \sqrt{5} t+(0.1+0.5 \cos \sqrt{3} t) e_{1}+0.7 e_{2} \sin 4 t \\
& +0.4 e_{3} \sin \sqrt{5} t+0.9 e_{12} \sin 5 t+(0.1+0.6 \sin \sqrt{7} t) e_{13} \\
& +\left(0.6 \cos 5 t+\frac{3}{10+t^{2}}\right) e_{23}+0.4 e_{123} \cos 3 t \\
& I_{2}(t)=0.7 e_{0} \sin \sqrt{3} t+0.6 e_{1} \cos 3 t+0.8 e_{2} \sin 6 t \\
& +0.8 e_{3} \cos \sqrt{3} t+0.5 e_{12} \sin 3 t+0.7 e_{13} \cos \sqrt{5} t \\
& +0.6 e_{23} \sin 3 t+0.9 e_{123} \cos 5 t+\frac{4}{20+t^{2}}, \\
& b_{11}(t)=0.03 e_{0} \sin 5 t+0.01 e_{3} \cos 2 t+0.01 e_{23} \sin 5 t+0.03 e_{123} \cos ^{2} 7 t \text {, } \\
& b_{12}(t)=0.04 e_{0} \cos 6 t+0.02 e_{2} \sin ^{2} 4 t+0.01 e_{3} \sin 3 t+0.03 e_{12} \cos 6 t \text {, } \\
& b_{21}(t)=0.025 e_{2} \sin 2 t+0.01 e_{3} \cos 3 t+0.02 e_{13} \cos 6 t+0.03 e_{23} \sin ^{2} 7 t, \\
& b_{22}(t)=0.04 e_{3} \cos 5 t+0.02 e_{12} \sin 7 t+0.02 e_{13} \cos ^{2} 6 t+0.03 e_{23} \cos 6 t \text {, } \\
& \tilde{b}_{11}(t)=0.02 e_{0} \cos t+0.01 e_{3} \cos 2 t+0.01 e_{23} \sin 4 t+0.03 e_{123} \sin ^{2} 5 t, \\
& \tilde{b}_{12}(t)=0.02 e_{0} \sin 3 t+0.02 e_{2} \cos ^{2} 4 t+0.01 e_{3} \cos 3 t+0.04 e_{12} \cos 3 t, \\
& \tilde{b}_{21}(t)=0.03 e_{2} \sin 5 t+0.01 e_{3} \cos 3 t+0.035 e_{13} \cos 4 t+0.02 e_{23} \sin ^{2} 7 t, \\
& \tilde{b}_{22}(t)=0.025 e_{3} \sin 3 t+0.01 e_{12} \sin ^{2} 5 t+0.04 e_{13} \cos 3 t+0.03 e_{23} \cos 2 t \text {, } \\
& \alpha_{11}(t)=\tilde{\alpha}_{11}(t)=0.01 e_{3} \cos 5 t+0.02 e_{12} \sin 3 t \\
& +0.02 e_{13} \sin ^{2} 6 t+0.01 e_{23} \sin 4 t \text {, } \\
& \alpha_{12}(t)=\tilde{\alpha}_{12}(t)=0.02 e_{2} \cos 4 t+0.04 e_{3} \sin 5 t \\
& +0.02 e_{12} \cos 4 t+0.04 e_{123} \cos 3 t, \\
& \alpha_{21}(t)=\tilde{\alpha}_{21}(t)=0.03 e_{1} \sin 3 t+0.02 e_{2} \cos 4 t \\
& +0.02 e_{3} \sin 5 t+0.01 e_{12} \cos ^{2} 2 t, \\
& \alpha_{22}(t)=\tilde{\alpha}_{22}(t)=0.05 e_{0} \sin 4 t+0.03 e_{1} \cos 5 t \\
& +0.02 e_{2} \cos 5 t+0.03 e_{13} \sin ^{2} 4 t \text {, } \\
& \beta_{11}(t)=\tilde{\beta}_{11}(t)=0.03 e_{0} \sin 2 t+0.01 e_{1} \cos 3 t \\
& +0.02 e_{2} \cos \sqrt{5} t+0.02 e_{123} \cos 3 t, \\
& \beta_{12}(t)=\tilde{\beta}_{12}(t)=0.02 e_{0} \cos 4 t+0.03 e_{1} \sin 5 t \\
& +0.04 e_{2} \cos \sqrt{6} t+0.01 e_{23} \sin 3 t, \\
& \beta_{21}(t)=\tilde{\beta}_{21}(t)=0.03 e_{0} \sin t+0.01 e_{1} \sin t \\
& +0.02 e_{2} \sin \sqrt{3} t+0.02 e_{12} \cos 4 t, \\
& \beta_{22}(t)=\tilde{\beta}_{22}(t)=0.015 e_{1} \cos 5 t+0.02 e_{2} \cos 3 t \\
& +0.01 e_{12} \sin \sqrt{6} t+0.02 e_{123} \cos 5 t,
\end{aligned}
$$




$$
\begin{aligned}
d_{i j}(t)= & 0.3 e_{0} \sin 2 \sqrt{5} t+0.3 e_{1} \cos 2 \sqrt{5} t \\
& +0.2 e_{12} \sin 2 t+0.1 e_{23} \sin \sqrt{2} t, \quad i, j=1,2, \\
T_{i j}(t)= & (0.2+0.1 \cos 2 t) e_{0}+0.4 e_{12} \sin ^{2} 3 t \\
& +0.6 e_{23} \sin 5 t+0.5 e_{123} \cos 4 t, \quad i, j=1,2, \\
S_{i j}(t)= & \left(0.5 \cos 6 t+\frac{3}{16+t^{2}}\right) e_{0}+0.4 e_{1} \sin 2 t \\
& +0.3 e_{2} \sin 5 t+0.4 e_{12} \cos t, \quad i, j=1,2, \\
\mu_{j}(t)= & 0.2 e_{2} \cos 2 t+0.2 e_{3} \cos ^{2} t+0.2 e_{23} \cos 3 t \\
& +\left(0.8 \sin \sqrt{3} t+\frac{2}{30+t^{2}}\right) e_{123}, \quad j=1,2 .
\end{aligned}
$$

If $\mathbb{T}=\mathbb{R}$, then we take

$$
\eta_{i j}(t)=0.4|\cos 3 t|, \quad \tau_{i j}(t)=0.7|\cos 6 t|, \quad \tilde{\tau}_{i j}(t)=0.9|\sin 3 t|, \quad i, j=1,2,
$$

and if $\mathbb{T}=\mathbb{Z}$, then we take

$$
\begin{aligned}
& \eta_{i j}(t)=3\left|\sin \left(3 \pi t+\frac{\pi}{2}\right)\right|, \quad \tau_{i j}(t)=2 e^{-5\left|\cos \left(\pi t+\frac{\pi}{2}\right)\right|}, \\
& \tilde{\tau}_{i j}(t)=4|\cos 4 \pi t|, \quad i, j=1,2 .
\end{aligned}
$$

Obviously, $\left(S_{1}\right)$ and $\left(S_{4}\right)$ hold. By calculating we have $L_{j}^{f}=\frac{1}{265}, L_{j}^{g}=L_{j}^{\tilde{g}}=\frac{3}{480}, L_{j}^{h}=L_{j}^{\tilde{h}}=\frac{\sqrt{15}}{1110}$, $j=1,2 ; a_{1}^{-}=0.35, a_{2}^{-}=0.4, c_{1}^{-}=0.6, c_{2}^{-}=0.3, b_{11}^{+}=\tilde{b}_{11}^{+}=b_{21}^{+}=\tilde{b}_{12}^{+}=0.03, b_{12}^{+}=b_{22}^{+}=\tilde{b}_{22}^{+}=$ $0.04, \tilde{b}_{21}^{+}=0.035, \alpha_{11}^{+}=\tilde{\alpha}_{11}^{+}=\beta_{22}^{+}=\tilde{\beta}_{22}^{+}=0.02, \alpha_{12}^{+}=\tilde{\alpha}_{12}^{+}=\beta_{12}^{+}=\tilde{\beta}_{12}^{+}=0.04, \alpha_{21}^{+}=\tilde{\alpha}_{21}^{+}=\beta_{11}^{+}=$ $\tilde{\beta}_{11}^{+}=\beta_{21}^{+}=\tilde{\beta}_{21}^{+}=0.03, \alpha_{22}^{+}=\tilde{\alpha}_{22}^{+}=0.05$;

$$
\begin{aligned}
& \mathcal{P}_{1} \approx 0.0963, \quad \mathcal{P}_{2} \approx 0.1165, \quad \mathcal{Q}_{1} \approx 0.0013, \\
& \mathcal{Q}_{2} \approx 0.0015, \quad \tilde{\mathcal{Q}}_{1} \approx 0.00179, \quad \tilde{\mathcal{Q}}_{2} \approx 0.00185, \\
& \max \left\{\frac{\mathcal{P}_{1}}{a_{1}^{-}}, \frac{\mathcal{P}_{2}}{a_{2}^{-}}\right\} \approx \max \{0.2751,0.2913\}=0.2913<\frac{1}{2}, \\
& \max \left\{\frac{\mathcal{Q}_{1}}{a_{1}^{-}}, \frac{\mathcal{Q}_{2}}{a_{2}^{-}}\right\} \approx \max \{0.0037,0.0038\}=0.0038=\kappa<1,
\end{aligned}
$$

and

$$
\max \left\{\frac{\tilde{\mathcal{Q}}_{1}}{a_{1}^{-}+c_{1}^{-}}, \frac{\tilde{\mathcal{Q}}_{2}}{a_{2}^{-}+c_{2}^{-}}\right\} \approx \max \{0.0019,0.0026\}=0.0026<1
$$

Hence, whether $\mathbb{T}=\mathbb{R}$ or $\mathbb{T}=\mathbb{Z}$, all the assumptions of Theorem 4.1 are satisfied. So by Theorem 4.1 systems (1) and (2) are globally exponentially synchronized about the pseudo almost periodic solution (see Figs. 1-10).

Figures 1 and 3 have the same initial values

$$
\left(x_{1}^{0}(0), x_{2}^{0}(0)\right)^{T}=\left(y_{1}^{0}(0), y_{2}^{0}(0)\right)^{T}=(0.2,-0.1)^{T},(1,-0.9)^{T},
$$



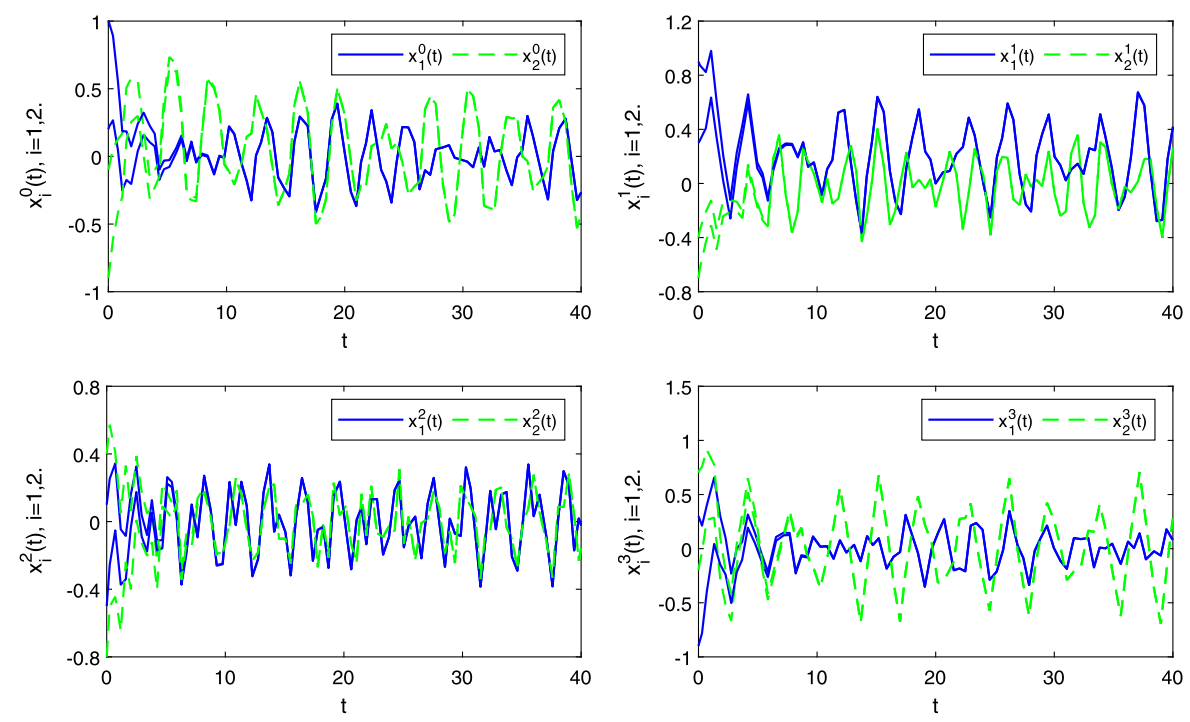

Figure $1 \mathbb{T}=\mathbb{R}$. Transient states of $x^{0}(t), x^{1}(t), x^{2}(t)$ and $x^{3}(t)$
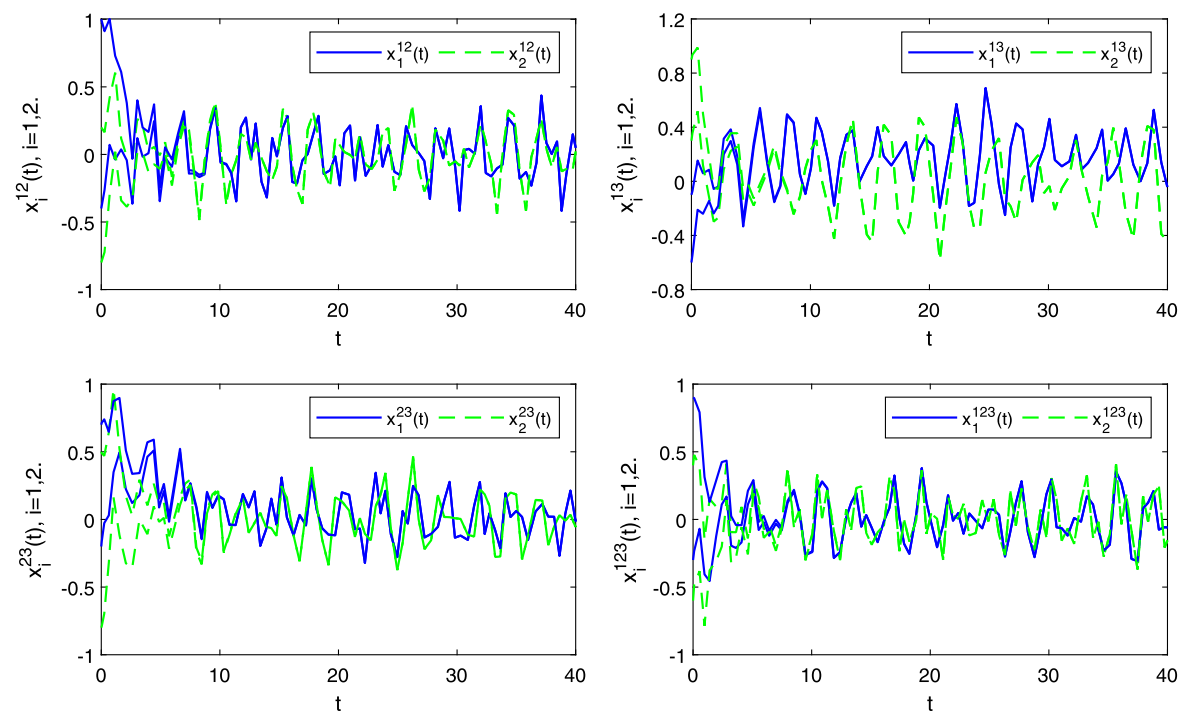

Figure $2 \mathbb{T}=\mathbb{R}$. Transient states of $x^{12}(t), x^{13}(t), x^{23}(t)$, and $x^{123}(t)$

$$
\begin{aligned}
& \left(x_{1}^{1}(0), x_{2}^{1}(0)\right)^{T}=\left(y_{1}^{1}(0), y_{2}^{1}(0)\right)^{T}=(0.3,-0.4)^{T},(0.9,-0.7)^{T}, \\
& \left(x_{1}^{2}(0), x_{2}^{2}(0)\right)^{T}=\left(y_{1}^{2}(0), y_{2}^{2}(0)\right)^{T}=(0.1,0.4)^{T},(-0.5,-0.8)^{T}, \\
& \left(x_{1}^{3}(0), x_{2}^{3}(0)\right)^{T}=\left(y_{1}^{3}(0), y_{2}^{3}(0)\right)^{T}=(0.3,-0.2)^{T},(-0.9,0.7)^{T} .
\end{aligned}
$$

Figures 2 and 4 have the same initial values

$$
\begin{aligned}
& \left(x_{1}^{12}(0), x_{2}^{12}(0)\right)^{T}=\left(y_{1}^{12}(0), y_{2}^{12}(0)\right)^{T}=(-0.4,0.2)^{T},(1,-0.8)^{T}, \\
& \left(x_{1}^{13}(0), x_{2}^{13}(0)\right)^{T}=\left(y_{1}^{13}(0), y_{2}^{13}(0)\right)^{T}=(-0.1,0.3)^{T},(-0.6,0.9)^{T},
\end{aligned}
$$



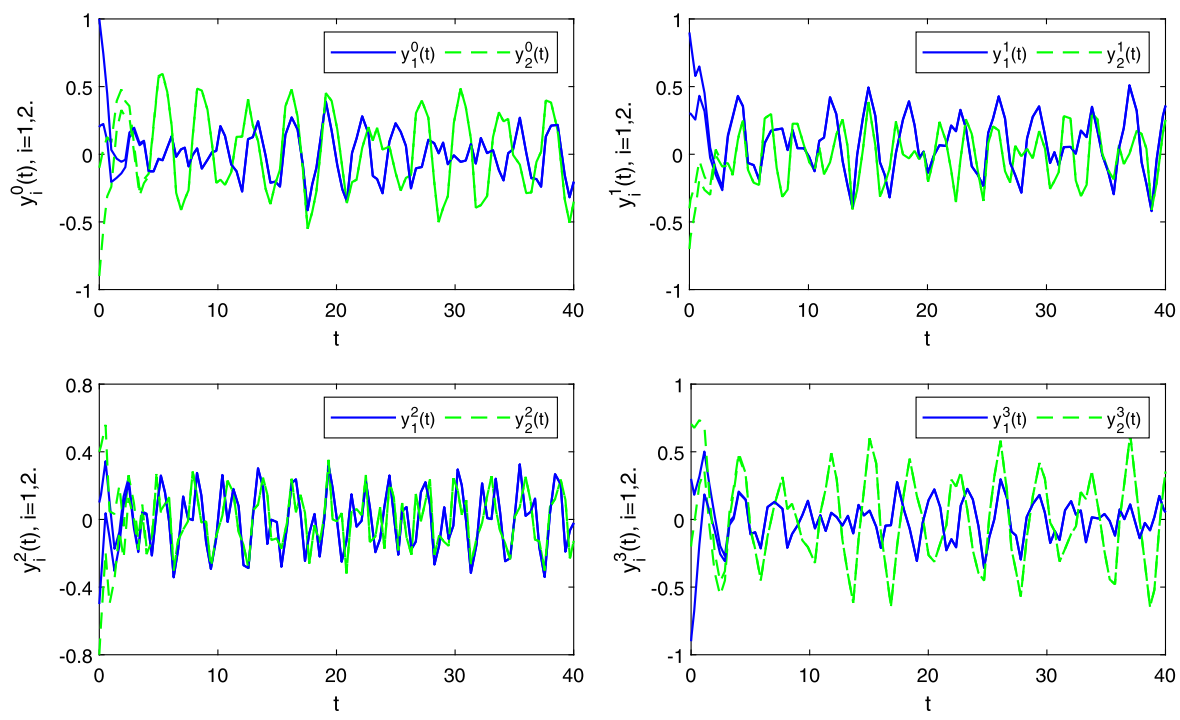

Figure $3 \mathbb{T}=\mathbb{R}$. Transient states of $y^{0}(t), y^{1}(t), y^{2}(t)$, and $y^{3}(t)$
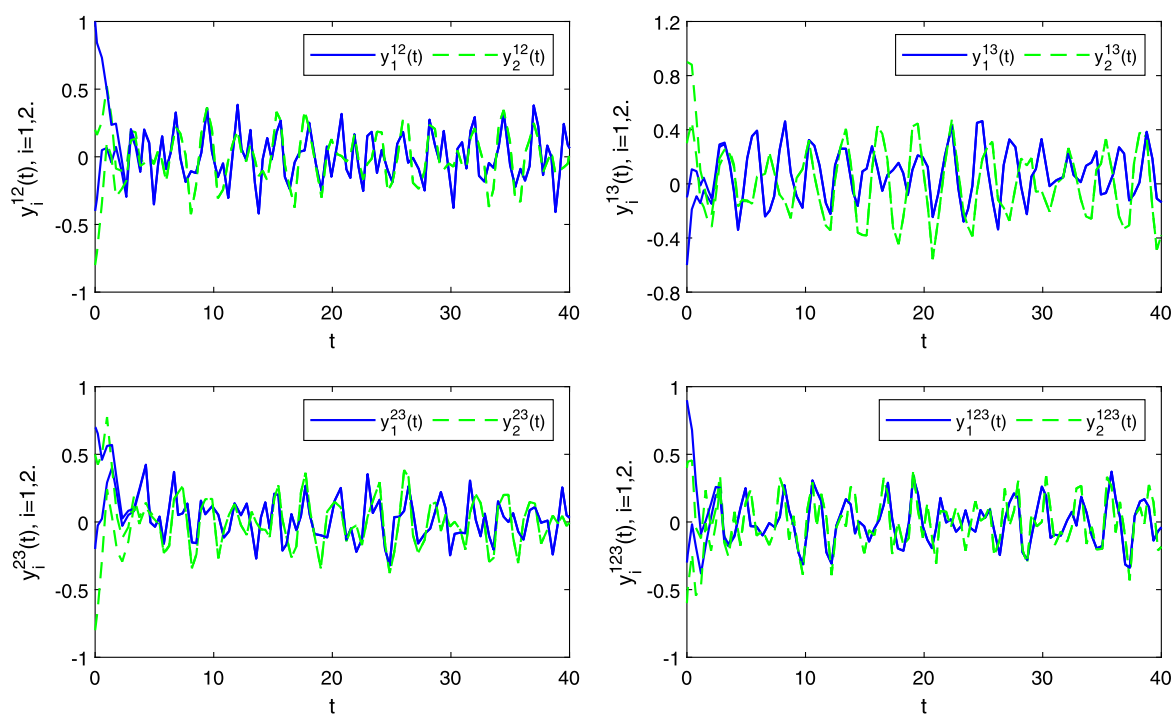

Figure $4 \mathbb{T}=\mathbb{R}$. Transient states of $y^{12}(t), y^{13}(t), y^{23}(t)$, and $y^{123}(t)$

$$
\begin{aligned}
& \left(x_{1}^{23}(0), x_{2}^{23}(0)\right)^{T}=\left(y_{1}^{23}(0), y_{2}^{23}(0)\right)^{T}=(-0.2,0.5)^{T},(0.7,-0.8)^{T}, \\
& \left(x_{1}^{123}(0), x_{2}^{123}(0)\right)^{T}=\left(y_{1}^{123}(0), y_{2}^{123}(0)\right)^{T}=(-0.3,0.4)^{T},(0.9,-0.6)^{T} .
\end{aligned}
$$

Figure 5 has two different initial values.

Figures 6 and 8 have the same initial values

$$
\begin{aligned}
& \left(x_{1}^{0}(0), x_{2}^{0}(0)\right)^{T}=\left(y_{1}^{0}(0), y_{2}^{0}(0)\right)^{T}=(0.5,-0.5)^{T},(-1,1)^{T}, \\
& \left(x_{1}^{1}(0), x_{2}^{1}(0)\right)^{T}=\left(y_{1}^{1}(0), y_{2}^{1}(0)\right)^{T}=(-0.7,0.6)^{T},(-1.2,1.5)^{T},
\end{aligned}
$$



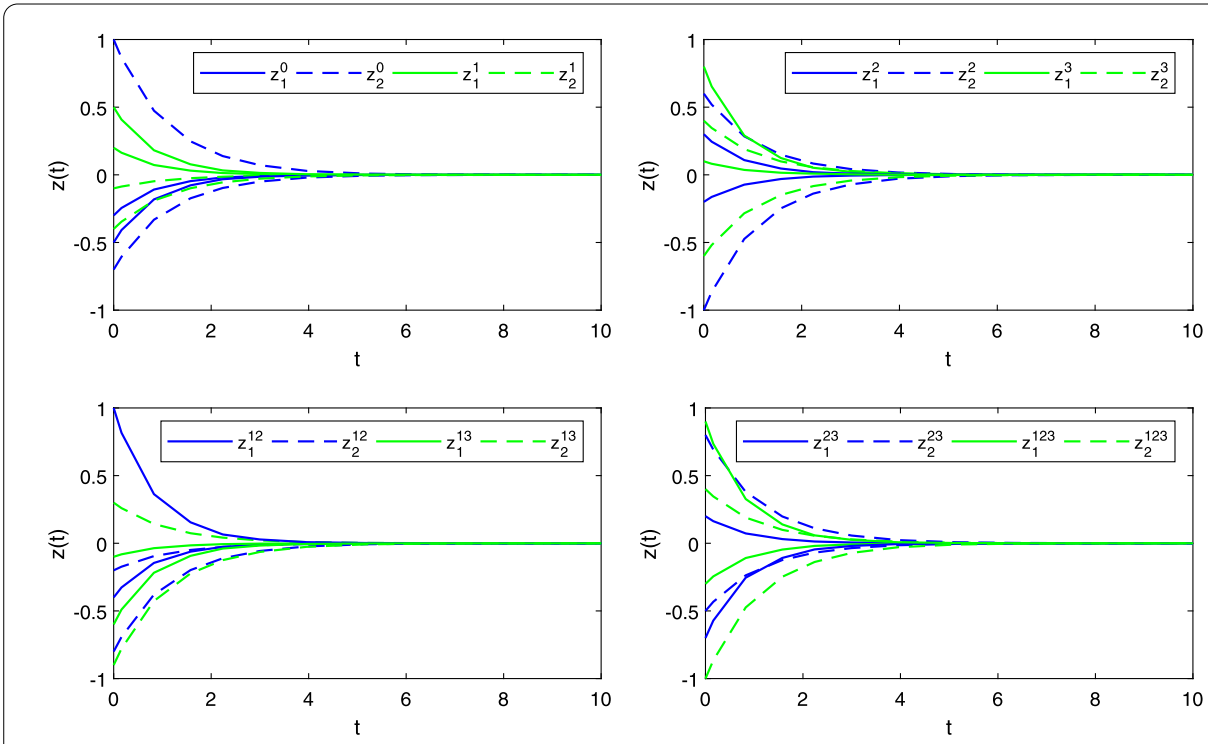

Figure $5 \mathbb{T}=\mathbb{R}$. Synchronization errors $z(t)=y(t)-x(t)$
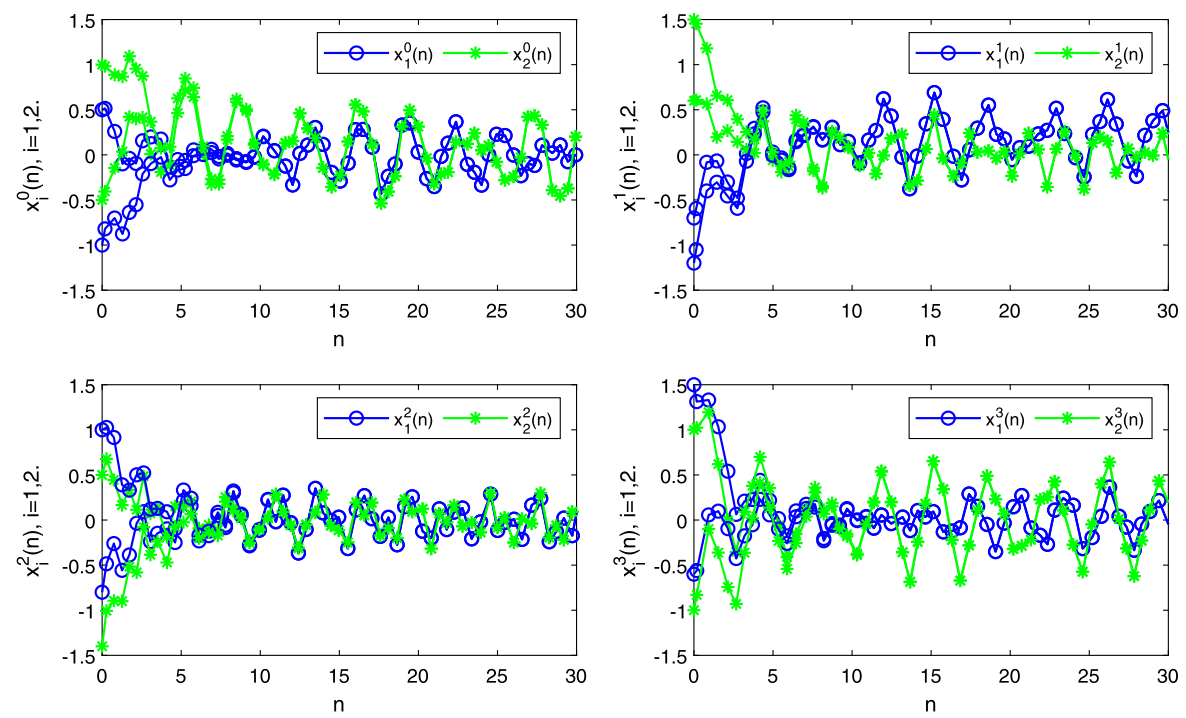

Figure $6 \mathbb{T}=\mathbb{Z}$. Transient states of $x^{0}(n), x^{1}(n), x^{2}(n)$ and $x^{3}(n)$

$$
\begin{aligned}
& \left(x_{1}^{2}(0), x_{2}^{2}(0)\right)^{T}=\left(y_{1}^{2}(0), y_{2}^{2}(0)\right)^{T}=(-0.8,0.5)^{T},(1,-1.4)^{T}, \\
& \left(x_{1}^{3}(0), x_{2}^{3}(0)\right)^{T}=\left(y_{1}^{3}(0), y_{2}^{3}(0)\right)^{T}=(1.5,1)^{T},(-0.6,-1)^{T} .
\end{aligned}
$$

Figures 7 and 9 have the same initial values

$$
\begin{aligned}
& \left(x_{1}^{12}(0), x_{2}^{12}(0)\right)^{T}=\left(y_{1}^{12}(0), y_{2}^{12}(0)\right)^{T}=(-1,0.8)^{T},(1.5,-1.5)^{T}, \\
& \left(x_{1}^{13}(0), x_{2}^{13}(0)\right)^{T}=\left(y_{1}^{13}(0), y_{2}^{13}(0)\right)^{T}=(1,-1.2)^{T},(-0.7,0.5)^{T}, \\
& \left(x_{1}^{23}(0), x_{2}^{23}(0)\right)^{T}=\left(y_{1}^{23}(0), y_{2}^{23}(0)\right)^{T}=(0.5,-1)^{T},(-1.5,1.3)^{T},
\end{aligned}
$$



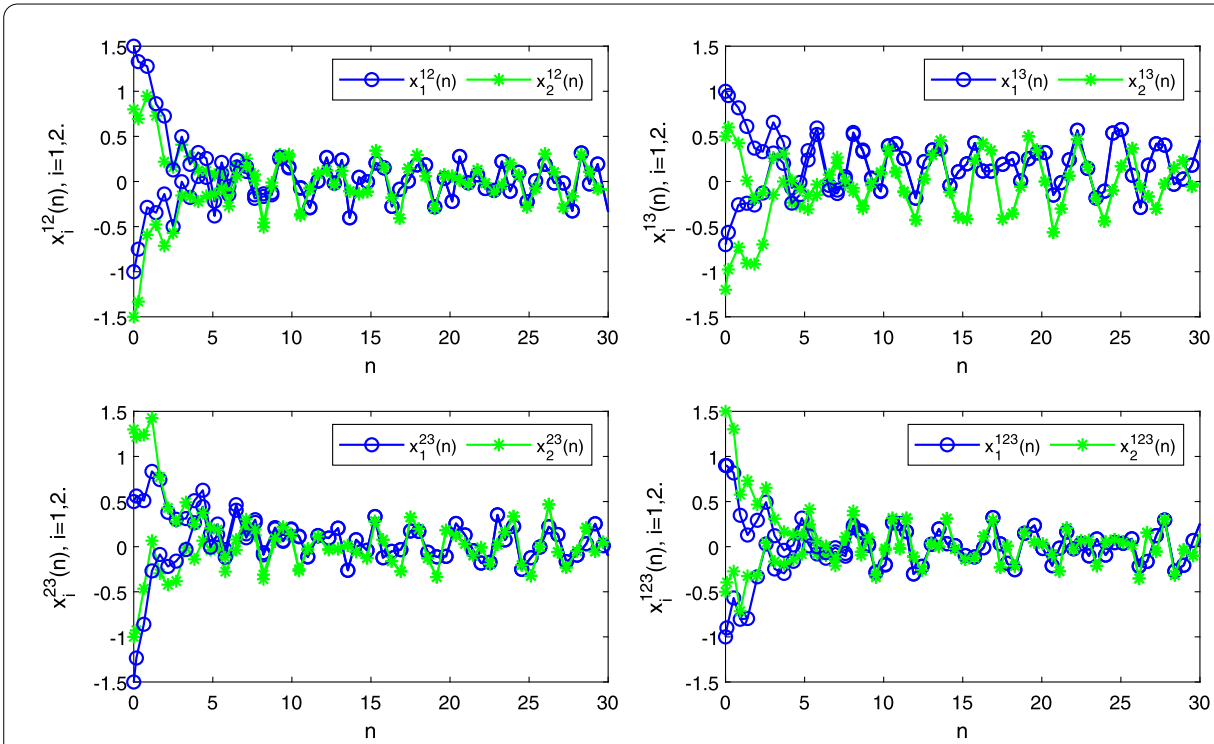

Figure $7 \mathbb{T}=\mathbb{Z}$. Transient states of $x^{12}(n), x^{13}(n), x^{23}(n)$, and $x^{123}(n)$
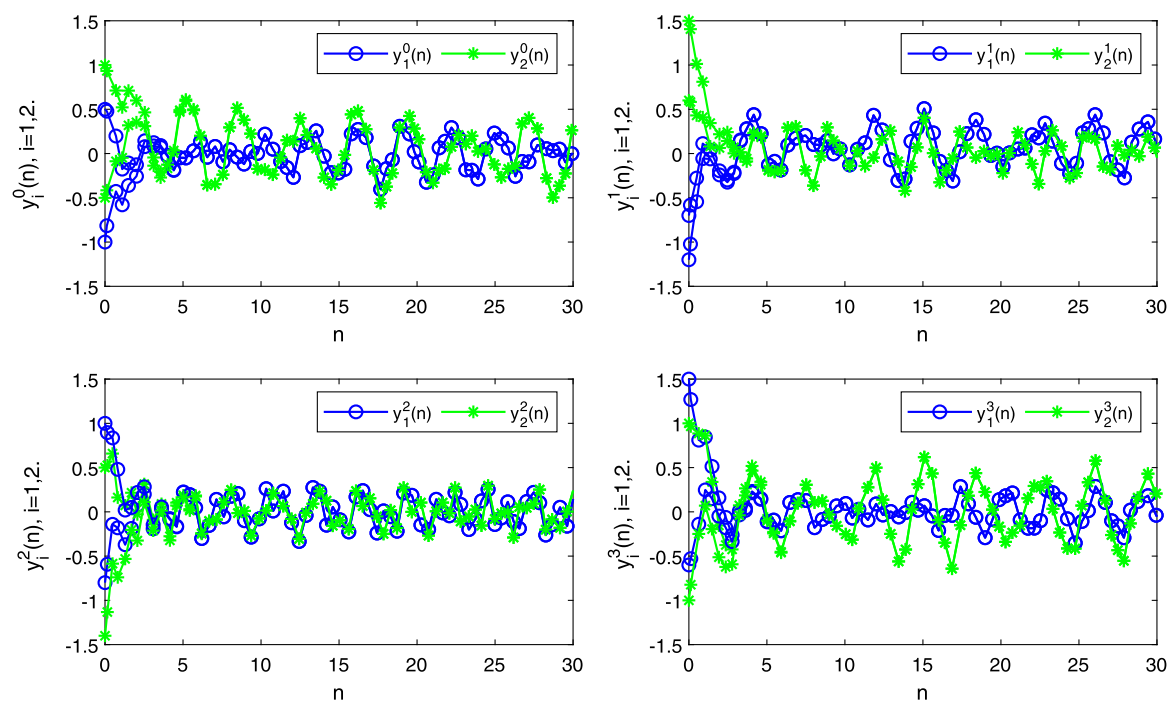

Figure $8 \mathbb{T}=\mathbb{Z}$. Transient states of $y^{0}(n), y^{1}(n), y^{2}(n)$, and $y^{3}(n)$

$$
\left(x_{1}^{123}(0), x_{2}^{123}(0)\right)^{T}=\left(y_{1}^{123}(0), y_{2}^{123}(0)\right)^{T}=(-1,-0.5)^{T},(0.9,1.5)^{T} .
$$

Figure 10 has two different initial values.

\section{Conclusion}

In this paper, we study pseudo almost periodic synchronization of Clifford-valued fuzzy cellular neural networks with time-varying delays on time scales by a direct method. That is to say, we do not decompose the Clifford-valued systems into real-valued systems, but directly study the Clifford systems. This is the first paper to study the pseudo almost periodic synchronization of Clifford-valued neural networks on time scales. The results of 

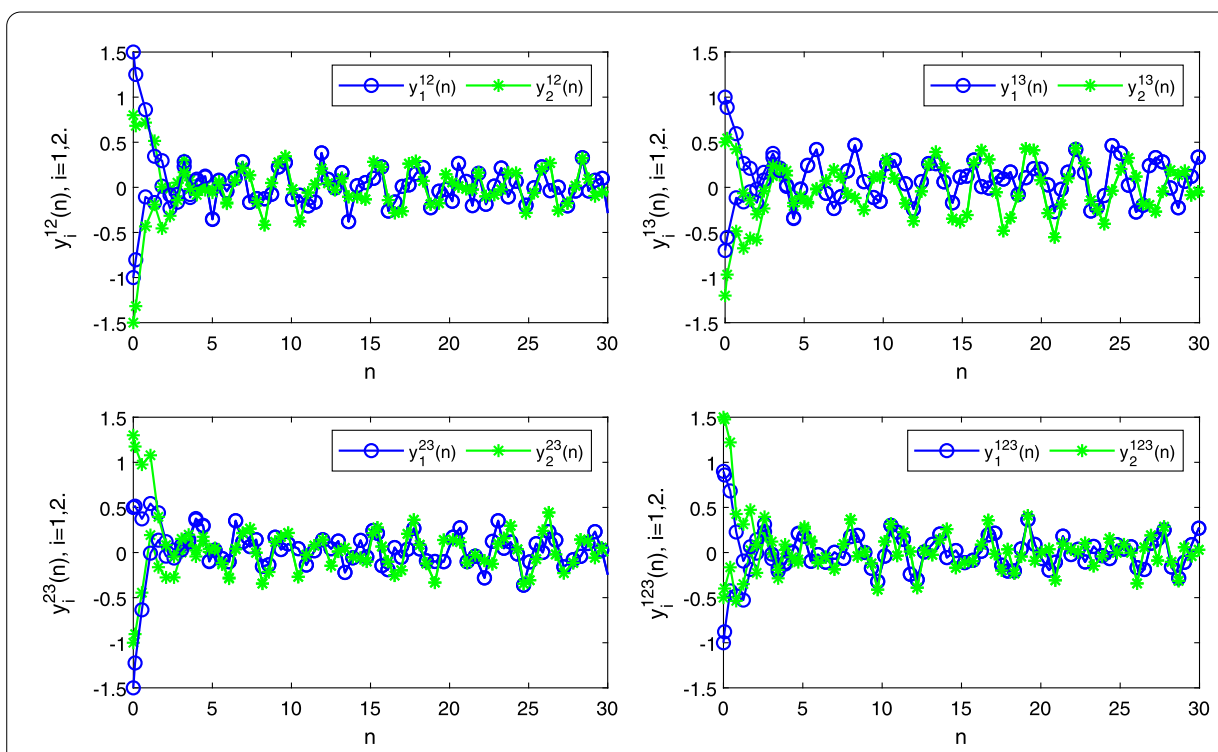

Figure $9 \mathbb{T}=\mathbb{Z}$. Transient states of $y^{12}(n), y^{13}(n), y^{23}(n)$, and $y^{123}(n)$
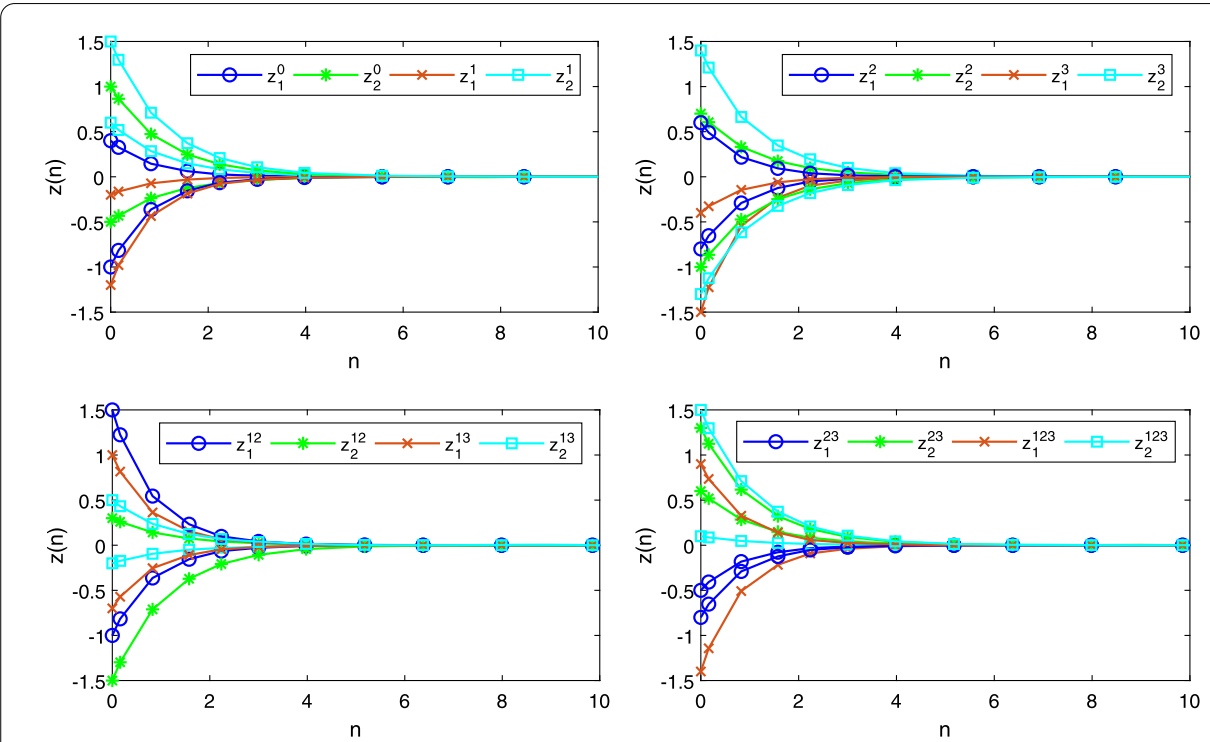

Figure $10 \mathbb{T}=\mathbb{Z}$. Synchronization errors $z(n)=y(n)-x(n)$

this paper are brand-new, and the proposed approach can be used to study the periodic, almost periodic, and almost automorphic synchronization for other types of neural networks on time scales. Studying the dynamics of Clifford-valued neural networks on time scales can not only unify the research of discrete- and continuous-time neural networks, but also unify the research of real-valued, complex-valued, and quaternion-valued neural networks. 
Funding

This work is supported by the National Natural Science Foundation of China under Grant 11861072.

\section{Availability of data and materials}

Data sharing not applicable to this paper as no datasets were generated or analyzed during the current study.

Ethics approval and consent to participate

Not applicable.

\section{Competing interests}

The authors declare that they have no competing interests.

Consent for publication

Not applicable.

Authors' contributions

Both authors contributed equally to the manuscript and typed, read, and approved the final manuscript.

\section{Publisher's Note}

Springer Nature remains neutral with regard to jurisdictional claims in published maps and institutional affiliations.

Received: 13 July 2020 Accepted: 8 October 2020 Published online: 21 October 2020

\section{References}

1. Yang, T., Yang, L.B., Wu, C.W., Chua, L.O.: Fuzzy cellular neural networks: theory. In: 1996 Fourth IEEE International Workshop on Cellular Neural Networks and Their Applications Proceedings (CNNA-96) IEEE, pp. 181-186 (1996)

2. Yang, T., Yang, L.B., Wu, C.W., Chua, L.O.: Fuzzy cellular neural networks: applications. In: 1996 Fourth IEEE International Workshop on Cellular Neural Networks and Their Applications Proceedings (CNNA-96) IEEE, pp. 225-230 (1996)

3. Abdurahman, A., Jiang, H., Teng, Z.: Finite-time synchronization for fuzzy cellular neural networks with time-varying delays. Fuzzy Sets Syst. 297, 96-111 (2016)

4. Huang, Z:: Almost periodic solutions for fuzzy cellular neural networks with multiproportional delays. Int. J. Mach Learn. Cybern. 8(4), 1323-1331 (2017)

5. Huang, Z:: Almost periodic solutions for fuzzy cellular neural networks with time-varying delays. Neural Comput. Appl. 28(8), 2313-2320 (2017)

6. Li, Y., Wang, C.: Existence and global exponential stability of equilibrium for discrete-time fuzzy BAM neural networks with variable delays and impulses. Fuzzy Sets Syst. 217, 62-79 (2013)

7. Jian, J., Wan, P.: Global exponential convergence of fuzzy complex-valued neural networks with time-varying delays and impulsive effects. Fuzzy Sets Syst. 338, 23-39 (2018)

8. Shen, S., Li, Y.: $S^{p}$-Almost periodic solutions of Clifford-valued fuzzy cellular neural networks with time-varying delays. Neural Process. Lett. 5(2), 1749-1769 (2020)

9. Duan, L., Wei, H., Huang, L.: Finite-time synchronization of delayed fuzzy cellular neural networks with discontinuous activations. Fuzzy Sets Syst. 361, 56-70 (2019)

10. Li, Y., Chen, X., Zhao, L.: Stability and existence of periodic solutions to delayed Cohen-Grossberg BAM neural networks with impulses on time scales. Neurocomputing 72(7-9), 1621-1630 (2009)

11. Li, Y., Meng, X., Xiong, L.: Pseudo almost periodic solutions for neutral type high-order Hopfield neural networks with mixed time-varying delays and leakage delays on time scales. Int. J. Mach. Learn. Cybern. 8(6), 1915-1927 (2017)

12. Lu, X.D., Zhang, X.: Stability analysis of switched systems on time scales with all modes unstable. Nonlinear Anal. Hybrid Syst. 33, 371-379 (2019)

13. Lu, X.D., Li, H.: An improved stability theorem for nonlinear systems on time scales with application to multi-agent systems. IEEE Trans. Circuits Syst. II (2020, in press). https://doi.org/10.1109/TCSII.2020.2983180

14. Lu, X.D., Li, H., Wang, C., Zhang, X.: Stability analysis of positive switched impulsive systems with delay on time scales. Int. J. Robust Nonlinear Control 30(16), 6879-6890 (2020)

15. Li, Y., Shen, S., Li, Y.: Almost automorphic solutions for Clifford-valued neutral-type fuzzy cellular neural networks with leakage delays on time scales. Neurocomputing 417, 23-35 (2020)

16. Shen, S., Li, Y.: Weighted pseudo almost periodic solutions for Clifford-valued neutral-type neural networks with leakage delays on time scales. Adv. Differ. Equ. 2020, 286, 24 pages (2020)

17. Pearson, J., Bisset, D.: Back propagation in a Clifford algebra. In: Aleksander, I., Taylor, J. (eds.) Artificial Neural Networks, pp. 413-416. North-Holland, Amsterdam (1992)

18. Pearson, J., Bisset, D.: Neural networks in the Clifford domain. In: IEEE International Conference on Neural Networks, Orlando, FL, USA, vol. 3, pp. 1465-1469 (1994)

19. Buchholz, S., Sommer, G.: On Clifford neurons and Clifford multi-layer perceptrons. Neural Netw. 21(7), 925-935 (2008)

20. Li, Y., Xiang, J.: Existence and global exponential stability of anti-periodic solution for Clifford-valued inertia Cohen-Grossberg neural networks with delays. Neurocomputing 332, 259-269 (2019)

21. Li, Y., Huo, N., Li, B.: On $\mu$-pseudo almost periodic solutions for Clifford-valued neutral type neural networks with delays in the leakage term. IEEE Trans. Neural Netw. Learn. Syst. (2020, in press). https://doi.org/10.1109/TNNLS.2020.2984655

22. Huo, N., Li, B., Li, Y.: Anti-periodic solutions for Clifford-valued high-order Hopfield neural networks with state-dependent and leakage delays. Int. J. Appl. Math. Comput. Sci. 30(1), 83-98 (2020)

23. Zhu, J.W., Sun, J.T.: Global exponential stability of Clifford-valued recurrent neural networks. Neurocomputing 173 685-689 (2016) 
24. Liu, Y., Xu, P., Lu, J.Q., Liang, J.L.: Global stability of Clifford-valued recurrent neural networks with time delays. Nonlinear Dyn. 84(2), 767-777 (2016)

25. Li, B., Li, Y.: Existence and global exponential stability of almost automorphic solution for Clifford-valued high-order Hopfield neural networks with leakage delays. Complexity 2019, Article ID 6751806 (2019)

26. Huo, N., Li, B., Li, Y.: Existence and exponential stability of anti-periodic solutions for inertial quaternion-valued high-order Hopfield neural networks with state-dependent delays. IEEE Access 7, 60010-60019 (2019)

27. Li, Y., Wang, Y., Li, B.: The existence and global exponential stability of $\mu$-pseudo almost periodic solutions of Clifford-valued semi-linear delay differential equations and an application. Adv. Appl. Clifford Algebras 29(5), 105, 18 pages (2019)

28. Liang, J., Qian, H., Liu, B.: Pseudo almost periodic solutions for fuzzy cellular neural networks with multi-proportional delays. Neural Process. Lett. 48(2), 1201-1212 (2018)

29. Liu, B:: Pseudo almost periodic solutions for neutral type CNNs with continuously distributed leakage delays. Neurocomputing 148, 445-454 (2015)

30. Liu, B.: Pseudo almost periodic solutions for CNNs with continuously distributed leakage delays. Neural Process. Lett. 42(1), 233-256 (2015)

31. Zhang, A.: Pseudo almost periodic solutions for SICNNs with oscillating leakage coefficients and complex deviating arguments. Neural Process. Lett. 45(1), 183-196 (2017)

32. Tang, Y.: Pseudo almost periodic shunting inhibitory cellular neural networks with multi-proportional delays. Neural Process. Lett. 48(1), 167-177 (2018)

33. Kong, F., Fang, X.: Pseudo almost periodic solutions of discrete-time neutral-type neural networks with delays. Appl. Intell. 48(10), 3332-3345 (2018)

34. Kong, F., Luo, Z., Wang, X.: Piecewise pseudo almost periodic solutions of generalized neutral-type neural networks with impulses and delays. Neural Process. Lett. 48(3), 1611-1631 (2018)

35. Tang, Y.: Exponential stability of pseudo almost periodic solutions for fuzzy cellular neural networks with time-varying delays. Neural Process. Lett. 49(2), 851-861 (2019)

36. Yang, G., Wan, W.: Weighted pseudo almost periodic solutions for cellular neural networks with multi-proportional delays. Neural Process. Lett. 49(3), 1125-1138 (2019)

37. Zhang, A.: Pseudo almost periodic high-order cellular neural networks with complex deviating arguments. Int. J. Mach. Learn. Cybern. 10(2), 301-309 (2019)

38. Arenas, A., Díaz-Guilera, A., Kurths, J., Moreno, Y., Zhou, C.: Synchronization in complex networks. Phys. Rep. 469(3), 93-153 (2008)

39. Wu, Z.G., Shi, P., Su, H., Chu, J.: Exponential synchronization of neural networks with discrete and distributed delays under time-varying sampling. IEEE Trans. Neural Netw. Learn. Syst. 23(9), 1368-1376 (2012)

40. Liu, X., Chen, T., Cao, J., Lu, W.: Dissipativity and quasi-synchronization for neural networks with discontinuous activations and parameter mismatches. Neural Netw. 24(10), 1013-1021 (2011)

41. Masoller, C., Zanette, D.H.: Anticipated synchronization in coupled chaotic maps with delays. Physica A 300(3-4), 359-366 (2001)

42. He, W.L., Cao, J.: Exponential synchronization of chaotic neural networks: a matrix measure approach. Nonlinear Dyn. 55(1-2), 55-65 (2009)

43. Chen, W.H., Luo, S.X., Zheng, W.X.: Impulsive synchronization of reaction-diffusion neural networks with mixed delays and its application to image encryption. IEEE Trans. Neural Netw. Learn. Syst. 27(12), 2696-2710 (2016)

44. Li, Y., Li, B., Yao, S.S., Xiong, L.L.: The global exponential pseudo almost periodic synchronization of quaternion-valued cellular neural networks with time-varying delays. Neurocomputing 303, 75-87 (2018)

45. Lu, X.D., Wang, Y., Zhao, Y.: Synchronization of complex dynamical networks on time scales via Wirtinger-based inequality. Neurocomputing 216, 143-149 (2016)

46. Lu, X.D., Zhang, X., Liu, Q.: Finite-time synchronization of nonlinear complex dynamical networks on time scales via pinning impulsive control. Neurocomputing 275, 2104-2110 (2018)

47. Rao, H., Liu, F., Peng, H., Xu, Y., Lu, R.: Observer-based impulsive synchronization for neural networks with uncertain exchanging information. IEEE Trans. Neural Netw. Learn. Syst. 31(10), 3777-3787 (2020)

48. Wang, L., Chen, T.: Finite-time and fixed-time anti-synchronization of neural networks with time-varying delays. Neurocomputing 329, 165-171 (2019)

49. Kong, F., Zhu, Q., Sakthivelc, R.: Finite-time and fixed-time synchronization control of fuzzy Cohen-Grossberg neural networks. Fuzzy Sets Syst. 394, 87-109 (2020)

50. Brackx, F., Delanghe, R., Sommen, F.: Clifford Analysis. Pitman Advanced Publishing Program, Boston (1982)

51. Bohner, M., Peterson, A.: Dynamic Equations on Time Scales, an Introduction with Applications. Birkhäuser, Boston (2001)

52. Li, Y., Wang, C.: Uniformly almost periodic functions and almost periodic solutions to dynamic equations on time scales. Abstr. Appl. Anal. 2011, Article ID 341520 (2011)

53. Li, B., Li, Y.: Existence and global exponential stability of pseudo almost periodic solution for Clifford-valued neutral high-order Hopfield neural networks with leakage delays. IEEE Access 7, 150213-150225 (2019) 